\title{
Stellenwert der ST-Arthroplastik in der Therapie der STT-Arthrose bei gleichzeitiger Rhizarthrose
}

Aus der Plastisch- und Handchirurgischen Klinik der Friedrich-Alexander-Universität Erlangen-Nürnberg Direktor: Prof. Dr. med. Dr. h. c. Raymund E. Horch

und der

Klinik für Plastische und Ästhetische Chirurgie Handchirurgie - Wiederherstellungschirurgie der St.-Antonius-Hospital gGmbH

Direktor: Priv.-Doz. Dr. med. Alexander Bach

Der Medizinischen Fakultät

der Friedrich-Alexander-Universität

Erlangen-Nürnberg

zur

Erlangung des Doktorgrades Dr. med. vorgelegt von

Maximilian Hermann Lorenz Halim 
Als Dissertation genehmigt von der

Medizinischen Fakultät der Friedrich-Alexander-Universität

Erlangen-Nürnberg

Vorsitzender des Promotionsorgans: Prof. Dr. Markus F. Neurath

Gutachter:

PD Dr. Alexander Daniel Bach

Gutachter:

Prof. Dr. Dr. Raymund Horch

Tag der mündlichen Prüfung:

25. Mai 2021 


\section{Widmung}

In Liebe und Dankbarkeit für meine Eltern Isolde \& Hermann Joseph, meine Geschwister Marie-Anne, Constantin, Cornelius und meine Freunde. 


\section{Inhaltsverzeichnis}

1. Einordnung in den fachwissenschaftlichen Kontext ..................................... 1

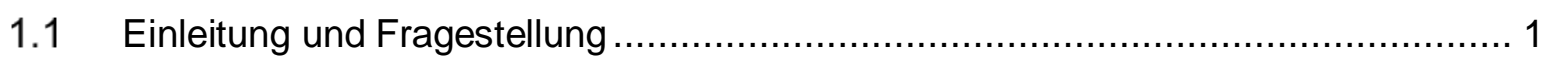

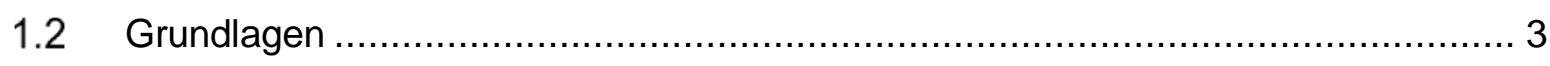

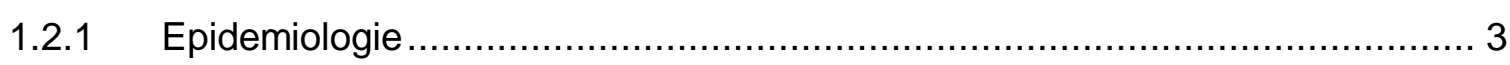

1.2.2 Ätiologie und Pathogenese ........................................................... 3

1.2.3 Relevante Anatomie und Biomechanik ................................................. 4

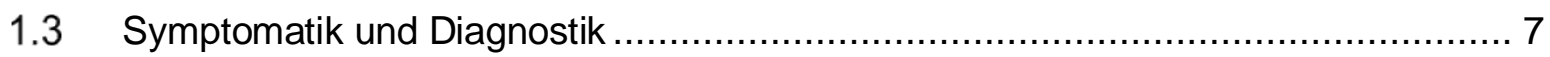

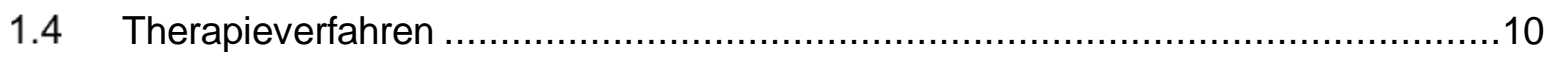

1.4.1 Nicht-operative Therapieverfahren.................................................... 10

1.4.2 Operative Therapieverfahren der Rhizarthrose ..................................... 11

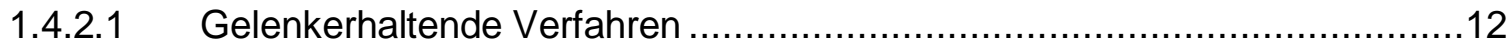

1.4.2.2 Gelenkresezierende Verfahren.................................................... 13

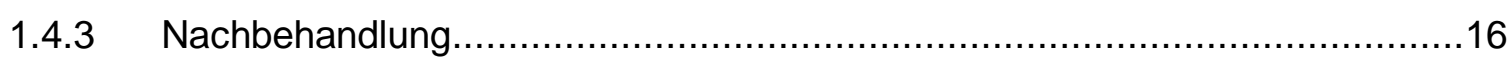

1.5 Material und Methoden der Nachuntersuchung .......................................17

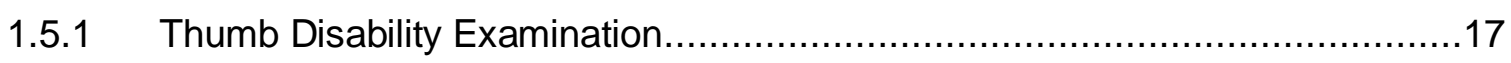

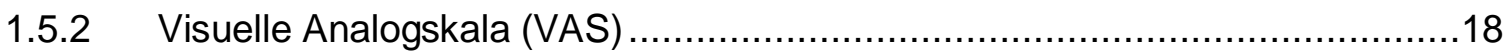

1.5.3 Funktionelle Klassifikation nach KAPANDJI / Kapandji-Score ..........................18

1.5.4 Kraftmessung mittels Martin-Vigorimeter..................................................18

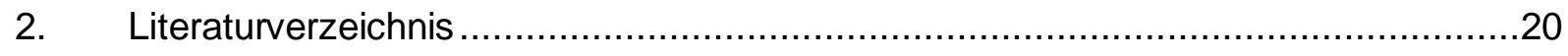

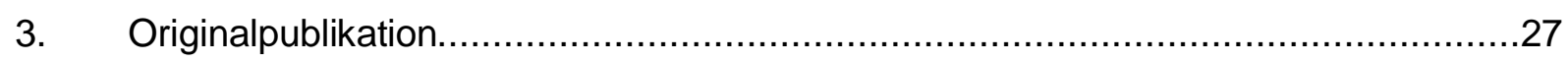

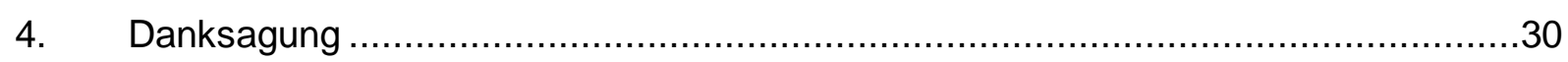




\section{Einordnung in den fachwissenschaftlichen Kontext}

\subsection{Einleitung und Fragestellung}

Der Daumen der menschlichen Hand und seine Fähigkeit zu opponieren hat evolutionsgeschichtlich höchste Relevanz für den Menschen. Die Fähigkeit, präzise zu greifen und zu halten, führte mitunter zur Erfindung und Nutzung von Instrumenten und Waffen, um einen deutlichen Überlebensvorteil und damit wesentlichen Unterschied zum Tierreich zu schaffen. Die Form und Funktion des Daumens ist für die Bewältigung alltäglicher Aufgaben von groBer Bedeutung. Dies ist daran zu erkennen, dass bei Verlust dieser Fähigkeit für Patienten erhebliche Probleme im Alltag entstehen und viele Gegenstände nur noch eingeschränkt nutzbar sind. Simple Tätigkeiten, wie das Öffnen einer Flasche, das Umdrehen eines Schlüssels oder das Schreiben mit einem Stift, sind ohne die Nutzung des Daumens plötzlich mit großen Schwierigkeiten und hohem Leidensdruck verbunden.

Die Daumensattelgelenkarthrose, die auch als Rhizarthrose [griechisch: ṕí $\alpha$ (rhiza) = Wurzel] bezeichnet wird, gehört zu den am häufigsten auftretenden Arthroseformen des menschlichen Bewegungsapparats und tritt insbesondere bei Frauen mittleren und höheren Alters auf (FORESTIER, 1937; WAGENHÄUSER, 1969; ARMSTRONG et al., 1994). Nicht selten entwickelt sich bei diesen Patienten zusätzlich eine Arthrose des skapho-trapezio-trapezoidalen Gelenkes (STT-Gelenk) (KATZEL et al., 2016).

Ab einem Lebensalter von über 55 Jahren weisen bis zu $65 \%$ der Bevölkerung radiologische Zeichen einer Rhizarthrose auf, jedoch nehmen viele Patienten nur gering ausgeprägte oder gar keine Symptome wahr (DAHAGHIN et al., 2005). Die Ursachen für die Entwicklung der Rhizarthrose sind mannigfaltig und es wird hauptsächlich zwischen idiopathischer, rheumatoider und posttraumatischer Arthrose unterschieden.

Der Daumen erhält seine charakteristische Fähigkeit zu opponieren durch die inkongruente und semirigide Form des Sattelgelenkes. Es wird angenommen, dass der Hauptrisikofaktor der idiopathischen Arthrose eine Überbelastung der Gelenkfläche bei gleichzeitiger Laxizität des Kapselbandapparates des Sattelgelenks ist (FONTANA et al., 2007).

Eine konservative Therapie findet vornehmlich Anwendung in frühen symptomarmen Stadien mit geringen Funktionsverlusten und bei geringem Leidensdruck der Patienten. Abhängig von der Schmerzsymptomatik, dem Leidensdruck und den Funktionsverlusten wird in späteren Stadien auf operative Verfahren zur Behandlung zurückgegriffen. Hierbei kommen viele verschiedene Operationsverfahren zur Anwendung, was ein Indiz für die Komplexität der 
Problematik ist, optimale, postoperative Ergebnisse in Bezug auf Schmerzen, Griffkraft, Stabilität und Bewegungsumfang zu erreichen (GANDER und WOLLSTEIN, 2013).

In manchen Fällen tritt die erhoffte Wiederherstellung der Funktion und Kraft sowie die Schmerzreduktion nicht ein oder die Symptomatik verschlechtert sich nach initialer Verbesserung sogar erneut. Ein Erklärungsansatz ist, dass eine begleitende oder sich im späteren Verlauf entwickelnde Arthrose des STT-Gelenkes dafür ursächlich ist (KATZEL et al., 2016; WARGANICH und SHIN, 2017).

Da sich bisher noch wenig in der Fachliteratur zur STT-Arthrose, ihrer Behandlung und insbesondere zum Patientenoutcome finden lässt, soll in dieser Arbeit untersucht werden, ob eine Sinnhaftigkeit darin besteht, bei Patienten, die sich einer operativen Therapie der Rhizarthrose unterziehen, gegebenenfalls eine Mitbehandlung des STT-Gelenkes durchzuführen. Dabei wird die Resektions-Suspensions-Arthroplastik (RSA) nach LUNDBORG, modifiziert nach WULLE, mit zusätzlicher Hemitrapezoidektomie, mit der konventionellen RSA ohne Hemitrapezoidektomie verglichen und die Ergebnisse der Nachuntersuchungen bewertet.

In den Jahren 2010 bis 2017 wurden in der Klinik für Plastische und Ästhetische Chirurgie Handchirurgie - Wiederherstellungschirurgie des Sankt-Antonius-Hospitals Eschweiler, Chefarzt: PD Dr. med. Alexander D. Bach, 539 Patienten mit Rhizarthrose unter Nutzung verschiedener Techniken (z. B. EPPING, WEILBY, LUNDBORG) operiert. Von diesen Patienten wurden 132 mittels konventioneller RSA nach LUNDBORG, modifiziert nach WULLE, und 205 mittels derselben Technik mit zusätzlicher Hemitrapezoidektomie operativ behandelt. Für die Studie wurden in der ersten Gruppe ohne Hemitrapezoidektomie 29 Patienten und 21 Patienten in der zweiten Gruppe mit Hemitrapezoidektomie nachuntersucht.

In der Nachuntersuchung der Patienten wurde zum Vergleich von posttherapeutischer Daumenfunktion, Schmerzen und Patientenzufriedenheit der TDX-Score (Thumb Disability Examination) erhoben. Die Schmerzintensität in Ruhe, bei leichter und intensiver Belastung wurde mittels visueller Analogskala (VAS) bestimmt. Die Oppositionsfähigkeit der Daumen wurde mittels Kapandji-Score gemessen und die Griffkraft mittels Martin-Vigorimeter ermittelt.

Die vorliegende Arbeit ist in zwei Teile untergliedert, von denen der erste der Einordnung in den wissenschaftlichen Kontext dient. Hierzu werden grundlegende relevante Themen in Bezug auf die Rhizarthrose und STT-Arthrose behandelt, anhand derer der Leser die im zweiten Teil aufgeführte Publikation einfacher nachvollziehen kann. 


\subsection{Grundlagen}

\subsubsection{Epidemiologie}

Die Daumensattelgelenkarthrose ist nach der distalen Interphalangealgelenk-Arthrose (DIP) die zweithäufigste Arthroseform der Finger (WILDER et al., 2006). Nach einer radiologischen Studie von S. DAHAGHIN et al. (2005) sollen bei $65 \%$ der Bevölkerung mit einem Lebensalter über 55 Jahren radiologische Zeichen einer Rhizarthrose nachweisbar sein. Hiervon sind insbesondere Frauen im postmenopausalen Alter mit einer bis zu 10 mal höheren Wahrscheinlichkeit betroffen als Männer im Vergleichsalter (MEIER et al., 2011; SWIGART, 2008). Die Prävalenz der symptomatischen Daumensattelgelenkarthrose bei Erwachsenen im Alter über 60 oder 70 Jahren liegt je nach Studie bei zwischen 1,9\% und 4,1 \% (KwOK et al., 2014). Da statistisch ermittelt in einer Bevölkerung mit überwiegend Rechtshändern das linke Daumensattelgelenk früher als das rechte von arthrotischen Vorgängen betroffen ist, wird davon ausgegangen, dass eine gut ausgeprägte Thenarmuskulatur protektiv dagegen wirkt (HAUGEN et al., 2011).

Das Vorliegen einer isolierten Arthrose des skapho-trapezio-trapezoidalen Gelenkes (STTGelenk) bei Frauen im postmenopausalen Alter liegt bei ungefähr 2 \% (ARMSTRONG et al., 1994). Die STT-Gelenk-Arthrose ist, nach der Arthrose im Gelenk zwischen Radius, Os scaphoideum und Os lunatum, die zweithäufigste im Bereich des Handgelenks (WATSON und BALLET, 1984). Besteht eine Rhizarthrose, so wird diese in $24 \%$ der Fälle von einer STTGelenk-Arthrose begleitet (KATZEL et al., 2016).

\subsection{2 Ätiologie und Pathogenese}

Bei der Entstehung der Rhizarthrose wird hauptsächlich zwischen idiopathischer (primär), rheumatoider und posttraumatischer (sekundär) Arthrose unterschieden.

Viele Ursachen der primären bzw. idiopathisch-degenerativen Rhizarthrose wurden in der Literatur diskutiert, konnten jedoch bisher nicht abschließend geklärt werden. Neben einem Alter über 40 Jahren, der Menopause, familiärer Belastung und beruflicher Exposition gilt mittlerweile insbesondere die weibliche Hormonkonstitution als Hauptrisikofaktor (JENSEN et al., 1999; RIYAZI et al., 2008; BOtHA-SCHEEPERS et al., 2009; ZHANG et al., 2009). Zudem begünstigen allgemeine Faktoren, wie angeborene oder erworbene Gelenkdysplasien, erhöhte Belastung durch Sport und Beruf, freie Gelenkkörper sowie neuromuskuläre Erkrankungen, die Entwicklung einer Arthrose durch eine erhöhte Druckbelastung auf den Gelenkknorpel (STÄBLER und WAGNER, 2014).

In häufigen Fällen ist das Daumensattelgelenk bei einer chronisch rheumatoiden Arthritis betroffen. Hierbei wird, wie auch bei anderen betroffenen Gelenken, der Knorpel durch 
autoimmune Prozesse von Makrophagen, Lymphozyten, Granulozyten, Osteoklasten und deren Entzündungsmediatoren und Enzymen angegriffen und destruiert (FASSBENDER, 1984).

Die posttraumatische Daumensattelgelenkarthrose entwickelt sich zumeist als Spätfolge aufgrund einer intraartikulären Fraktur des Os trapezium oder der Metakarpale-I-Basis, wie der Bennett- oder Rolando-Fraktur. Da sich die präzise Reposition der Gelenkflächen bei Gelenkfrakturen ohne Stufenbildung schwierig gestaltet und gelegentlich nicht gänzlich gelingt, entwickelt sich konsekutiv im Langzeitverlauf häufig eine Rhizarthrose.

Allgemein kann gesagt werden, dass sich durch Verschleißprozesse die korrespondierenden Knorpelflächen zunehmend abnutzen. Sobald kein Knorpel mehr als Gleitfläche vorhanden ist, besteht unmittelbarer Knochenkontakt, der bei Bewegungen Schmerzen auslöst. Da sich durch die Abnahme der Knorpelmasse folglich der Gelenkspalt verschmälert, sinkt ebenso die Spannung der Gelenkkapsel und die Laxizität nimmt zu. Durch die erhöhte Bewegungsfreiheit entwickelt sich im Verlauf eine pathologische Arthrokinematik, die zunächst zur Subluxation des MHK I und im späteren Stadium durch Osteophytenbildung zu einer Bewegungseinschränkung im Gelenk führt sowie final zu einer Gelenksteife mit fixierter Fehlstellung im Sinne eines „Pollex adductus“ führen kann (MERLE und VOCHE, 2009; PELLEGRINI, 2001).

Die Ursachen für die Entwicklung einer STT-Arthrose sind ebenfalls vielfältig. So kann sie unter anderem in Folge einer Band-Verletzung der Handwurzel, einer Fraktur, einer Chondrokalzinose oder rheumatoiden Arthritis entstehen. Zudem begünstigt die durch eine Rhizarthrose entstehende Fehlbelastung des STT-Gelenks die Entwicklung einer Arthrose (RICHTER, 2015).

\subsubsection{Relevante Anatomie und Biomechanik}

Das Daumensattelgelenk (Articulatio carpometacarpalis pollicis, CMC-I-Gelenk oder auch Trapeziometakarpalgelenk I) wird von den artikulierenden Gelenkflächen der Basis des Os metacarpale I und des Os trapezium gebildet. Die Gelenkfläche des Os trapezium erhält durch die in dorso-palmarer Richtung konvexe und in radio-ulnarer Richtung konkave Biegung ihr sattelförmiges und damit namensgebendes Aussehen. Die Gelenkfläche des Os trapezium ist schmaler und abgeflachter als die Gelenkfläche des Os metacarpale I (ZUMHASCH et al., 2012). Hierbei unterscheidet sich die Anatomie des weiblichen Daumensattelgelenks von der männlichen durch flachere Krümmungsradien, kleinere Gelenkflächen und einen dünneren Knorpelbelag (NORTH und RUTLEDGE, 1983; ATESHIAN et al., 1992; XU et al., 1998). Diese Inkongruenz bedingt nur in der Hälfte der Bewegungen eine Deckungsgleichheit der Gelenkflächen, sodass ein vollständiger Gelenkverschluss nicht möglich ist. In 
endgradiger Abduktions- oder Adduktionsstellung liegt maximale Kongruenz vor (MERLE und VOCHE, 2009). Das Daumensattelgelenk hat, ähnlich einem Kugelgelenk, drei Freiheitsgrade: Durch seine Form ermöglicht es die Bewegung des Daumens in der Flexions-/Extensionsachse, der Abduktions-/Adduktionsachse und erhält bei Opposition durch die Rotationsbewegung des 1. Mittelhandknochens um die axiale Längsachse den dritten Freiheitsgrad (SCHMIDT und LANZ, 2013a). Im Sattelgelenk beträgt der Umfang der Adduktion ca. 25 Grad, der Abduktion 35 Grad, der Flexion ca. 25 Grad, Extension ca. 45 Grad und Rotation ca. 10 Grad (ZUMHASCH et al., 2012). Der Bewegungsumfang der Opposition beträgt 10-15 Grad und der der Retroposition 40-60 Grad (SCHMITT und HAHN, 2014).

Insbesondere bei der Opposition wirken punktuell hohe Scherkräfte, sowohl am Randbereich der palmaren und dorsalen Gelenkflächen des Os metacarpale I als auch an den artikulierenden Flächen des Os trapezium (RICHTER, 2015). COONEY und CHAO entdeckten, dass bei Anwendung des Pinzettengriffs zwischen Daumen und Zeigefinger mit einer Last von $1 \mathrm{~kg}$ im Durchschnitt im Interphalangealgelenk $3 \mathrm{~kg}$, im Daumengrundgelenk 5,4 kg und im Sattelgelenk $12 \mathrm{~kg}$ wirken (COONEY und CHAO, 1977).

Das Daumensattelgelenk besitzt physiologisch eine schlaffe und sehr weite Gelenkkapsel, die im Zusammenspiel mit der geringen Gelenkführung den großen Bewegungsumfang ermöglicht. Die Stabilisierung des Gelenkes erfolgt durch primäre (ligamentäre) und sekundäre (muskuläre) Stabilisatoren. Rund um das Sattelgelenk gibt es insgesamt 16 kapsuläre und extrakapsuläre Bänder, von denen keines im Einzelnen für die Stabilität des Gelenkes verantwortlich ist (BETTINGER et al., 1999; BetTINGER und BERGER, 2001). Es existiert keine Position des Gelenkes, in der alle Bänder gleichzeitig entspannt sind (PIERON, 1973; IMAEDA et al., 1993). Von den 16 Bändern haben insbesondere sieben Ligamente gemeinsam einen wesentlichen Einfluss auf die Stabilität des Gelenkes und laut verschiedener Autoren auch unterschiedliche Bedeutung für die Entstehung der Rhizarthrose: das Ligamentum (Lig.) carpometacarpale obliquum anterius superficiale, das Lig. carpometacarpale obliquum anterius profundum, das Lig. carpometacarpale obliquum posterius, das Lig. intermetacarpale, das Lig. intermetacarpale dorsale, das Lig. carpometacarpale dorsoradiale und das Lig. collaterale ulnare (BETTINGER et al., 1999; HORCH und UNGLAUB, 2011). Das Lig. carpometacarpale dorsoradiale und das Lig. carpometacarpale posterius, welche gemeinsam den dorsalen Bandkomplex bilden, sowie die intermetakarpalen Ligamente zwischen dem Os metacarpale I und II gelten heute als die für die Stabilität relevantesten Bänder (BETTINGER et al., 2000; Colman et al., 2007; EdMUNDS, 2011; NANNO et al., 2017; TAN et al., 2011).

Sowohl die Thenarmuskulatur (intrinsic muscles) als auch die langen Daumenmuskeln mit Ursprung am Unterarm (extrinsic muscles) sind für die Gelenkstabilität des Daumensattelgelenks wichtig. Zu diesen gehören der Musculus (M.) interosseus dorsalis I, M. opponens 
pollicis, M. adductor pollicis sowie die Musculi (Mm.) extensores pollicis, Mm. flexores pollicis und Mm. abductores pollicis (HORCH und UNGLAUB, 2011).

Das skapho-trapezio-trapezoidale Gelenk bildet die radiale Säule der artikulierenden Übergänge von der proximalen zur distalen Reihe der Handwurzelknochen. Das Gelenk wird aus der distal-radialen konvexen Gelenkfläche des Os scaphoideum und proximalen konkaven Gelenkflächen des Os trapezium und des Os trapezoideum gebildet (SCHMIDT und LANZ, 2013b). Die Stabilisierung des STT-Gelenkes erfolgt über ein komplexes System aus Bändern, Kapselbändern und Muskeln. Der ligamentäre STT-Komplex besteht aus vier Komponenten: einem starken Lig. scaphotrapezium, dem Lig. scaphocapitatum, einer schwachen palmaren und zarten dorsalen Kapsel (DREWNIANY et al., 1985). Die durch eine Rhizarthrose entstehende Fehlbelastung des dorsoradialen Trapeziumrandes kann zu einer Rotation des Os trapezium führen, was eine unphysiologische Kraftverteilung für die Artikulationsflächen des Os scaphoideum und Os trapezoideum zur Folge hat und die Entstehung einer Pantrapezialarthrose begünstigen kann (RICHTER, 2015). 


\subsection{Symptomatik und Diagnostik}

Die Diagnose einer Rhizarthrose wird mittels anamnestischer Befragung, klinischer Untersuchung und röntgentechnischer Bildgebung gestellt.

Als Kardinalsymptom der Rhizarthrose gilt die Schmerzsymptomatik, die für jeden Patienten individuell und abhängig vom Stadium der Arthrose ist. Die Patienten geben typischerweise an, im Alltag Schwierigkeiten beim Öffnen einer Flasche mit Drehverschluss sowie beim Greifen und Hochheben einer Flasche zu haben. In den Anfangsstadien zeigen sich äußerlich keine Auffälligkeiten im Bereich des Daumensattelgelenks. Hierbei ist die Opposition und Adduktion nur unter Belastung schmerzhaft (EATON und GLICKEL, 1987; GLICKEL, 2001).

In fortgeschrittenen Stadien nimmt die Schmerzsymptomatik mit häufiger Projektion in das Handgelenk zu. Diese tritt nun nicht mehr nur morgens und in Kombination mit Morgensteife auf, sondern entwickelt sich bis hin zu starkem Ruheschmerz. Äußerlich lässt sich meist eine Schwellung, Rötung und Überwärmung als Zeichen einer (re-)aktivierten Arthrose sowie eine M- bzw. Z-förmige Fehlstellung des Daumensattelgelenks im Sinne einer Subluxation feststellen. Das auftretende Forestier-Zeichen beschreibt die sekundäre Hyperextension des Daumengrundgelenkes, die sich durch die Zugwirkung des $\mathrm{M}$. adductor pollicis longus bei gleichzeitiger Kapselbandlaxizität entwickelt, sodass bei progredientem, langjährigem Verlauf eine zunehmende Parallelstellung des MHK I zum MHK II zu beobachten ist (MERLE und VOCHE, 2009). Plötzlich auftretende Schmerzen und zunehmende Kraftlosigkeit führen dazu, Gegenstände nicht mehr in der betroffenen Hand halten zu können.

Die Knochendichte des Os metacarpale I und die Thenarmuskulatur atrophieren aufgrund von Schonung und Inaktivität im Verlauf. Das Bewegungsausmaß des Daumens wird durch die arthrotischen Prozesse zunehmend bis hin zur fixierten Subluxation eingeschränkt. Die Schmerzen und Gelenksteife nehmen zu, sodass sich im Endstadium eine Gebrauchsunfähigkeit der Hand zeigen kann.

In der klinischen Untersuchung zeigt sich die kräftige Palpation des Daumensattelgelenks schmerzhaft. Beim „Grind-Test“ (Reibe-Test) wird durch den Untersucher eine axiale Stauchung auf den I. Strahl bei simultaner Zirkumduktion ausgeübt, während im „Crank-Test“ (Verdreh-Test) der axial gestauchte Daumen passiv flektiert und extendiert wird (STÄBLER und WAGNER, 2014). Stadienabhängig können so durch beide Tests Schmerzen sowie hörund spürbare Krepitationen ausgelöst werden (SWANSON, 1972; MERRITT et al., 2010). Der „Distraktions-Rotations-Test“ bedient sich des Prinzips des „Grind-Tests“, wobei hier jedoch ein axialer Zug auf den I. Strahl ausgeübt wird und somit Schmerzen provoziert werden können. Der Repositionstest wird als positiv gewertet, sobald der nach ventro-lateral gerichtete Druck auf das bereits subluxierte Daumensattelgelenk Schmerzen im Gelenk verursacht 
(HORCH und UNGLAUB, 2011). Bei gleichzeitiger STT-Arthrose entstehen bei passiver Bewegung im Handgelenk insbesondere bei Flexion/Extension in Radialduktion zusätzliche Schmerzen (RICHTER, 2015).

Besteht nach der Anamnese und klinischen Untersuchung der Verdacht einer Rhizarthrose, erfolgt eine röntgenologische Darstellung des Daumensattelgelenks im posterior-anterioren und seitlichen Strahlengang zur Stadieneinteilung. Zur besseren Beurteilung werden die Spezialprojektionen „Daumen palmodorsal und seitlich“ mit Zentrierung auf das Sattelgelenk empfohlen (SCHMITT und HAHN, 2014). Die röntgenologische Klassifizierung nach EATON und LITTLER unterteilt die radiologisch dargestellten Veränderungen des Gelenkes in vier Stadien:

Im Stadium I zeigen sich normale Gelenkkonturen bei einem erweiterten Gelenkspalt.

Im Stadium II sind eine geringe Gelenkspaltverschmälerung, sklerotische und zystische Veränderungen mit Osteophyten oder freien Gelenkkörpern mit einer Größe bis zu 2 Millimeter erkennbar.

Im Stadium III zeigen sich eine fortgeschrittene Gelenkspaltverschmälerung, sklerotische und zystische Veränderungen mit Osteophyten oder freien Gelenkkörpern mit einer Größe von über 2 Millimeter.

Im Stadium IV stellen sich zusätzlich zu den degenerativen Veränderungen des Daumensattelgelenks im Stadium III auch solche im STT-Gelenk dar (EATON et al., 1984; EATON und GLICKEL, 1987).

Die typischen Arthrosezeichen zeigen sich jedoch stärker am Os trapezium und den Peritrapezialgelenken als am Os metacarpale I (STÄBLER und WAGNER, 2014).

Da manche Röntgenbilder eine stark ausgeprägte Symptomatik vermuten lassen, die Patienten jedoch kaum Schmerzen verspüren, gilt in der Therapie der Rhizarthrose insbesondere der Leitspruch „Patienten behandeln, nicht Röntgenbilder“ (GLICKEL, 2001).

Die Rolle von CT und MRT sowie Sonographie spielen in der Diagnostik einer Rhizarthrose eine eher untergeordnete Rolle und können im besten Falle in frühen Stadien diagnostisch und im Verlauf zur Planung von Implantaten oder Korrektionsosteotomien dienlich sein.

Als Differenzialdiagnosen müssen stets eine Tendovaginitis stenosans de Quervain, Styloiditis radii, ein Karpaltunnelsyndrom, eine aseptische Os-lunatum-Knochennekrose, eine Ringbandstenose sowie andere degenerative, traumatische oder entzündliche Prozesse umliegender Strukturen, wie z.B. SL-Bandverletzungen, SNAC- oder SLAC-Wrist oder Skaphoidpseudarthrosen, berücksichtigt und ausgeschlossen werden (DEILER, 2019; HORCH 
und UNGLAUB, 2011). Insbesondere durch einen positiven Grind-Test und einen negativen Finkelstein-Test kann eine Tendovaginitis stenosans de Quervain klinisch zumeist ausgeschlossen werden (RICHTER, 2015). In bis zu 43 \% der Fälle einer Rhizarthrose kann ein Karpaltunnelsyndrom gleichzeitig bestehen und daher in derselben Operation durch Spaltung des Retinaculum flexorum behandelt werden (FLORACK et al., 1992).

In der klinischen Untersuchung ist das STT-Gelenk von dorsal in der Vertiefung zwischen den Sehnen des M. extensor carpi radialis brevis und M. extensor pollicis longus ca. zwei Fingerbreit distal des Tuberculum dorsale tastbar. Bei auslösbarem Druckschmerz gilt der STT-Test als positiv und es sollte weiterführende Diagnostik durchgeführt werden (FRANK, 2015). Radiologisch kann das STT-Gelenk am besten mittels der Zitherspieler-Schrägaufnahme im konventionellen Röntgen beurteilt werden (SCHMITT und UNGLAUB, 2014). Die Röntgenzeichen einer STT-Arthrose sind die vermehrte subchondrale Sklerose, meist fehlende oder nur gering ausgeprägte osteophytäre Ausziehungen, ein sich vergrößernder $\mathrm{Ge}$ lenkspalt bei der hypermobilen STT-Arthrose während der Ulnarduktion sowie konkave Auswalzung der Gelenkfläche des Os trapezium im Seitenbild. Liegt eine blockierte STT-Arthrose vor, zeigt sich der Gelenkspalt verschmälert und das Os scaphoideum zusätzlich in Extensionsstellung (STÄBLER und WAGNER, 2014). 


\subsection{Therapieverfahren}

\subsubsection{Nicht-operative Therapieverfahren}

Die degenerativen und strukturellen Veränderungen einer Arthrose sind irreversibel. Daher kann durch konservative Therapien höchstens eine Verlangsamung des Verschleißes erwirkt werden. Im Stadium I und II nach EATON und LITTLER und bei noch schwach ausgeprägter Symptomatik wird zumeist konservativ therapiert. Das oberste Ziel ist hierbei vor allem die Schmerzlinderung (WOLF und DeLARONDE, 2012; YAO und PARK, 2008).

Mittels einer Daumenorthese kann eine temporäre Ruhigstellung des Daumensattelgelenks erwirkt und damit die Schmerzen, insbesondere in den Anfangsstadien I und II, in bis zu 76 \% der Fälle gelindert werden (SWIGART et al., 1999; MADDALI-BONGI et al., 2016; BUHLER et al., 2019). Da die Schmerzlinderung nur durch das konsequente Tragen der Schiene erwirkt werden kann, setzt dies eine hohe Patientencompliance und -akzeptanz voraus, was sich im Alltag bei einer Tragedauer von 16 Stunden für mindestens 30 Tage jedoch häufig als therapie-erschwerender Faktor darstellen kann (COLDITZ, 2000).

Eine Kryotherapie findet Anwendung bei akut auftretenden Schmerzen im Daumensattelgelenk. Dahingegen können dem Patienten bei chronischem Verlauf lokale oberflächliche und tieferdringende Wärmeanwendungen Linderung verschaffen. Zudem haben sich Ultraschall und Niedrigfrequenzelektrotherapie in Form von diadynamischen Strömen als unterstützend erwiesen (CASIMIRO et al., 2002; EBENBICHLER, 2009).

Als Optionen der Strahlenbehandlung stehen eine protrahiert fraktionierte Schmerzbestrahlung sowie eine Radiosynoviorthese zur Verfügung. Bei der Schmerzbestrahlung erfolgt die Bestrahlung mittels Linearbeschleuniger zwei Mal wöchentlich für drei Wochen mit jeweils 1 Gray (KALTENBORN et al., 2016).

Bei der Radiosynoviorthese erfolgt durch eine intraartikuläre Injektion eines Radionuklids eine Destruktion oberflächlicher, entzündeter Anteile der Synovia ohne darunter liegende Knorpelstrukturen zu beschädigen. Je nach erwünschter Strahlendosis werden entweder Ytrrium-90, Rhenium-186 oder Erbium-169 zur Therapie verwendet. Nachdem die Radionuklide durch die Synovialzellen phagozytiert wurden, entstehen dort Nekrosen durch die Strahlenreaktion. Im weiteren Verlauf folgt eine Fibrosierung und Sklerosierung der Synovialis und somit der Rückgang der Entzündung. Als unerwünschte Begleiterscheinungen können neben möglichen Infektionen auch periartikuläre Nekrosen und Verklebungen benachbarter Strukturen entstehen, welche eine Bewegungseinschränkung und im weiteren Verlauf eine Verkomplizierung einer operativen Therapie nach sich ziehen können (GABRIEL et al., 2019). 
Röntgenologisch kontrollierte, intraartikuläre Injektionen von Kortikosteroiden oder Hyaluronsäure, gegebenenfalls in Kombination mit Lokalanästhetika, können bei Patienten mit Arthrose in niedrigen Stadien zu kurzfristiger Schmerzlinderung führen, wobei eine langfristige Linderung noch umstritten ist (DAY et al., 2004; FOWLER et al., 2015; KROON et al., 2016). Eine zeitgleiche Behandlung mittels Daumensattelgelenkorthese kann die Chancen einer Linderung jedoch ergänzend erhöhen.

Als weitere Optionen der Behandlung im frühen Stadium stehen auch physiotherapeutische und ergotherapeutische Maßnahmen zur Verfügung und können sich positiv auf die Schmerzen, Beweglichkeit und Funktion auswirken (DAVENPORT et al. 2012; VILLAFAÑE et al., 2012; ØSTERÅS et al., 2013).

Für die medikamentöse antiphlogistische Therapie werden zeitweise Cyclooxygenase-2Hemmer und nichtsteroidale Antirheumatika (NSAR) als orale Medikation genutzt. Die Potenz der Schmerzreduktion ist jedoch nur moderat (GRIFKA et al., 2004). Zudem können auch topische Antiphlogistika in Kombination mit immobilisierenden Verbänden unterstützend wirken.

\subsubsection{Operative Therapieverfahren der Rhizarthrose}

Bei unzureichendem Erfolg der konservativen Therapie nach 3 Monaten, bei fortgeschrittener Symptomatik und in späteren Stadien gelten die verschiedenen operativen Therapieverfahren als letzte Möglichkeit, um eine Schmerzlinderung und einen Funktionserhalt der Hand zu erwirken (BAKER et al., 2017). Ausschlaggebend für eine OP-Indikationsstellung sind hier primär die klinische Darstellung und der Leidensdruck der Patienten, da bis heute noch keine objektiven Kriterien für die Art und den Zeitpunkt der Operation festgelegt werden konnten. Aufgrund der hohen Komplexität des Erkrankungsbildes, der damit einhergehenden individuellen Ausprägung der Symptomatik sowie der operateur-abhängigen Vorlieben für verschiedene Methoden ist die Datenlage zu dem Thema sehr unterschiedlich. Dadurch können bisher keine klaren Aussagen zur eindeutigen Überlegenheit einer einzelnen Operationstechnik gegenüber den anderen Methoden getroffen werden (VERMEULEN et al., 2011; WAJON et al., 2017).

Die operativen Verfahren können grob in gelenkerhaltende und gelenkresezierende Verfahren eingeteilt werden. In früheren Stadien finden, wenn überhaupt, gelenkerhaltende Verfahren Anwendung, während ab Stadium III in der Regel auf gelenkresezierende Operationen zurückgegriffen wird.

Die Arthroskopie besitzt eine besondere Stellung in der Therapie, da sie nicht nur diagnostische, sondern auch simultan therapeutische Möglichkeiten unter minimalinvasiven Bedingungen offenhält. So kann sie zur Evaluation des Knorpels, der Bänder und deren Stabilität 
sowie des Entzündungsprozesses angewendet werden (BERGER, 1997). Ferner reichen die Therapiemöglichkeiten mittlerweile von gelenkerhaltenden Verfahren, wie Debridement, Synovektomie und Bandstraffung mittels Radiofrequenz-Ablation durch thermisches Kapselshrinking oder Inlay-Implantationen, bis hin zu gelenkresezierenden Therapien, wie der partiellen oder gänzlichen Trapezektomie, Resektionsions-Suspensionsarthroplastiken mit möglicher zeitgleicher Behandlung einer STT-Arthrose (ABZUG und OSTERMAN, 2011; BADIA, 2007; CobB et al., 2011; EdWARDS und RAMSEY, 2010; MENON, 1996).

\subsubsection{Gelenkerhaltende Verfahren}

Die gelenkerhaltenden Verfahren finden hauptsächlich im Stadium I bis teilweise II nach EATON und LITTLER bei schwach ausgeprägter Symptomatik, alleiniger Rhizarthrose und hauptsächlicher Instabilität des Gelenkes Anwendung. Hierzu gehören Verfahren wie die Denervation nach WILHELM, Umstellungsosteotomien, Kapselbandplastiken oder Debridements. Ziel dieser Verfahren ist, die Progression der Rhizarthrose zu vermeiden respektive zu verlangsamen, um zu einem späteren Zeitpunkt im Sinne einer Therapieeskalation auf gelenkresezierende Verfahren zurückgreifen zu können.

\section{Denervation}

Nach erfolgreicher Testblockade werden bei der Denervation nach WILHELM die sensiblen gelenkinnervierenden Äste des Nervus (N.) medianus und N. radialis operativ ausgeschaltet. Hierbei werden die Äste des N. digitalis dorsalis proprius I und Ramus (R.) articularis r. palmaris $n$. mediani, sowie die des $R$. superficialis $n$. radialis, des $R$. articularis spatii interossei I und des R. articularis n. cutaneus antebrachii radialis mittels Elektrokauter durchtrennt (WILHELM, 1972, 1966). Vorteile der Denervierung sind eine geringe Invasivität, eine kurze Nachbehandlungsdauer, niedrige Kosten sowie die Option der Therapieeskalation in Richtung gelenkresezierender Verfahren ohne eine Beeinträchtigung des OP-Gebietes (LORÉA, 2003). Die Erfolgsrate kann bis zu 81 \% betragen (FouCHER et al., 1998).

\section{Umstellungsosteotomie des Os metacarpale I und des Os trapezium}

Durch die Umstellungsosteotomie des Os metacarpale I nach WILSON soll die Fehlbelastung der Gelenkflächen mittels dorsal schließender Keilosteotomie (closing wedge) der Basis des Metakarpale I korrigiert werden (WILSON, 1973). Seit 1973 konnte durch die Entwicklung verschiedener Modifikationen in Studien gezeigt werden, dass die Osteotomie zumindest einen partiell korrektiven biomechanischen Effekt haben kann und auch hierbei eine Therapieeskalation zu intraartikulären Verfahren möglich bleibt (MIURA et al., 2004; PARKER et al., 2008; PELLEGRINI et al., 1996; TOMAINO, 2011). Als alternative Technik dazu kann eine öffnende Osteotomie (open wedge) des Os trapezium dienen, bei der von dorsoradial eine Inzision in das Os trapezium vorgenommen und der Spalt bis zur gewünschten Korrektur sukzessive aufgebogen wird (CHEEMA et al., 2012). 


\section{Kapsel-Band-Plastiken}

Kapsel-Band-Plastiken können bei geringem Knorpelschaden die Gelenkstabilität bei subluxiertem Daumensattelgelenk wiederherstellen und sollen präventiv die Progredienz der Rhizarthrose verzögern. Die 1973 erstmals beschriebene Bandplastik nach EATON-LITTLER nutzt einen distal gestielten Sehnenstreifen des M. flexor carpi radialis (FCR-Sehne), um den palmaren Bandkomplex direkt und den dorsalen indirekt zu verstärken (EATON und LITTLER, 1973). Bei dieser OP-Technik wird der Sehnenstreifen zunächst durch die Basis des Os metacarpale I von palmar nach dorsal, dann tief unter dem Ansatz der Sehne des M. abductor pollicis longus (APL-Sehne) hindurch und zuletzt um den verbliebenen intakten FCR-Sehnenanteil herumgeführt. Dort wird er unter Zug mit der APL-Sehne vernäht (EATON et al., 1984). In Anlehnung an dieses Prinzip wurden Techniken entwickelt, die FCR-Sehnenanteile zur Verstärkung der dorsalen und interkarpalen Bänder oder einen Teil der APL-Sehne zu nutzen (BRUNELLI et al., 1989; RUST und THAM, 2011).

\subsubsection{Gelenkresezierende Verfahren}

Es gibt eine Vielzahl gelenkresezierender Verfahren und auch diese werden, jeweils die individuelle Situation des Patienten berücksichtigend, ab einem Stadium II ohne zusätzliche arthrotische Beteiligung des STT-Gelenks angewendet. Viele dieser Methoden ähneln sich und verfolgen jeweils dasselbe Ziel: die Verbesserung oder zumindest den Erhalt der Funktion und Kraft des Daumens bei simultaner Beschwerdefreiheit. Zu den Verfahren gehören die alleinige Trapezektomie, Pyrocarbon- und Gelenkprothesenimplantationen, die Arthrodese des trapeziometakarpalen Gelenks, Mini TightRope ${ }^{\circledR}$ Suspensionen sowie die verschiedenen Arten von Resektions-Suspensions-Interpositionsarthroplastiken (BARRERAOCHOA et al., 2014; CHUG et al., 2014; MARICONDA et al., 2014; SCHIBLI-BEER et al., 2008; SZALAY et al., 2014, 2011, 2009; VERMEULEN et al., 2011).

\section{Trapezektomie}

Bei der Trapezektomie, welche die Grundlage für die zahlreichen Modifikationen der Resektions-Suspensions-Arthroplastik und andere Methoden bildet, erfolgt die alleinige Entfernung des Os trapezium; sie wurde erstmals 1948 von GERVIS beschrieben (GERVIS, 1948). Diese Technik wird angewendet, sofern der erhaltene Kapsel-Band-Apparat des Sattelgelenks nach Entfernung des Os trapezium kräftig erscheint, eine ausreichende Stabilität und keine deutliche Schädigung aufweist. Bei ausreichender Stabilität des Bandapparates bleibt der Daumen postoperativ bei funktioneller Belastung in der nahezu physiologischen Position.

\section{Resektions-Suspensions-Arthroplastik (RSA)}

Liegt eine Instabilität der interkarpalen Bandstrukturen des Sattelgelenks vor, hervorgerufen durch Elongation oder Risse der Bänder aufgrund entzündlicher oder traumatischer 
Prozesse, kommt es häufig zur Proximalisierung des Os metacarpale I, was eine häufige postoperative Komplikation darstellt (COONEY et al., 2006). Hierbei entsteht ein Kontakt zwischen der Basis des Os metacarpale I und der distalen Gelenkfläche des Os scaphoideum, welcher erneut zu Schmerzen und Funktionseinschränkungen führt. Um dies zu verhindern, stehen dem Operateur einerseits eine zusätzliche Kapselplastik, wie oben beschrieben, und andererseits eine Suspensionsarthroplastik des Os metacarpale I mittels Sehnenumleitung oder Implantat als weiterführende Therapiemöglichkeiten zur Verfügung. Bei der Trapezektomie mit Kapselplastik wird das Kapsel-Band-Gewebe genutzt, um durch Straffung eine stabile Aufhängung zu erwirken.

Die Resektions-Suspensions-Arthroplastik (RSA) gilt zurzeit als Goldstandard der Therapie und es gibt einige modifizierte Verfahren, von denen die RSA nach EPPING und nach LUNDBORG und deren jeweilige weiteren Modifikationen die populärsten darstellen.

Bei der RSA nach EPPING wird nach der Entfernung des Os trapezium die Sehne des M. flexor carpi radialis (FCR) unter Erhalt des Sehnenansatzes an der Basis des Os metacarpale II longitudinal halbiert. Durch eine ca. 6-10 cm vom Sehnenansatz entfernte Querinzision entsteht ein freier Sehnenstreifen. Die Basis des Os metacarpale I wird quer durchbohrt und der freie Sehnenanteil von medial nach lateral hindurchgeführt und mit sich selbst vernäht (EPPING, 1989).

Bei der von LUNDBORG beschriebenen Technik wird die Sehne des M. abductor pollicis longus (APL) auf ähnliche Weise wie bei der Methode nach EPPING gespalten. Dieser wird durch einen Tunnel sowohl in der Sehne des M. flexor carpi radialis als auch in dem verbliebenen anheftenden Teil der Sehne des M. abductor pollicis longus selbst gezogen und jenseits dessen mit sich selbst vernäht (SIGFUSSON und LUNDBORG, 1991). Bei der Modifikation nach WULLE wird der freie Sehnenanteil entweder durch die FCR-Sehne gezogen oder um sie herum gelegt und danach direkt mit sich selbst vernäht, um ein breiteres Netz als Aufhängeplastik zu schaffen (WULLE, 1993). Bei allen beschriebenen Techniken kann der restliche Anteil des freien Sehnenanteils im Sinne einer Interposition in den Raum zwischen Os metacarpale und Os scaphoideum eingelegt werden, um dort als Platzhalter respektive Polster zu fungieren.

Statt autologes Sehnenmaterial für die Suspension zu nutzen, besteht die Möglichkeit künstliche Materialien wie das Mini TightRope ${ }^{\circledR}$ zu verwenden. Hierbei wird, nach Bohrung von querverlaufenden Kanälen durch die Basis des MHK I und MHK II, das Mini TightRope® durch diese hindurchgefädelt, aufgespannt und fixiert. Diese Technik scheint nach aktueller Datenlage jedoch eine Proximalisierung des 1. Mittelhandknochens nach Trapezektomie nicht sicher verhindern zu können und stellt daher aufgrund von schmaler Studienlage zur 
Zeit lediglich eine Therapieoption und keine Empfehlung dar (SZALAY et al., 2015; BRAUN et al., 2016).

\section{Prothetik des Daumensattelgelenks}

Anstelle des oben genannten freien Sehnenanteils kann ein Allotransplantat-Platzhalter, wie

z. B. ein künstlicher Silastik-Spacer, als Trapeziumersatz eingelegt werden. Diese Technik hat jedoch nach einstweiligem klinischen Erfolg aufgrund von Langzeitkomplikationen, wie Entzündungen der Gelenkkapsel, Prothesensubluxationen und knöchernen Auffälligkeiten, an Bedeutung in der Behandlung der Rhizarthrose verloren (ZSCHÖCK-HOLLE et al., 2015).

Die Auswahl an Miniprothesen, durch die das Daumensattelgelenk ersetzt werden kann, ist groß. Die kugelgelenkartigen Totalendoprothesen können zementfrei oder zementiert implantiert werden. Die endoprothetische Versorgung scheint zwar in Bezug auf Kraft, Bewegungsumfang und Schmerzfreiheit der Resektions-Suspensions-Arthroplastiken überlegen zu sein, jedoch stellen, neben hohen Raten von Prothesenlockerungen, v. a. Infektionen, Frakturen und Ausrenkungen Komplikationen dar, die zu Reoperationen führen (CEBRIANGOMEZ et al., 2019). Derzeit fehlen noch aussagekräftige Langzeituntersuchungen, sodass eine endgültige Aussage zur Einordnung der verschiedenen Modelle im Kontext der Rhizarthrose bisher nicht getätigt werden kann.

\section{Arthrodese des Daumensattelgelenks}

Durch die Versteifung des Daumensattelgelenks soll für den Patienten eine für Dauerbelastungen stabile und schmerzfreie Funktionalität des Daumens erzielt werden. Die Kraft kann hierbei nahezu vollkommen erhalten bleiben; jedoch wird hierdurch der Bewegungsumfang des Daumens deutlich verringert und die Präzision der Griffe sinkt. Dem Operateur stehen für die Fixation zwischen dem Os metacarpale I und dem Os trapezium verschiedene Techniken mit unterschiedlichen Osteosynthesematerialen, wie z.B. Kirschner-Drähte, Cerclagen, Herbert-Schrauben, Platten u. a., zur Verfügung (MERLE und VoCHE, 2009). Die Arthrodese des Sattelgelenks bringt ein erhöhtes Risiko für die Entwicklung einer STT-Arthrose mit sich, da die Scherkräfte direkt vom Daumen weitergeleitet werden und die benachbarten Gelenke einer inadäquat hohen Belastung aussetzen. Im Vergleich zu Resektionsarthroplastiken liegt eine erhöhte Komplikations- und Reoperationsrate aufgrund von Beschwerden durch Pseudarthrosenbildung (8-21\%), Anschlussarthrosen oder Problemen mit dem Osteosynthesematerial vor (TAYLOR et al., 2005; RAVEN et al., 2007; VERMEULEN et al., 2014). Aufgrund der oben genannten Gründe wird die Arthrodese heute zumeist nur noch bei jüngeren Patienten mit posttraumatischer Rhizarthrose und hohem Anspruch an Stabilität unter extremer Belastung angewendet. 


\section{Behandlung einer begleitenden STT-Arthrose}

Die begleitende oder sich im späteren Verlauf entwickelnde Arthrose des STT-Gelenks wird in Kombination mit den oben genannten Techniken mittels Hemitrapezoidektomie operativ therapiert. Hierbei wird einzeitig oder im Sinne einer Reoperation das Os trapezoideum in der Mitte quer gespalten und der proximale Anteil reseziert. In die entstandene Resektionshöhle kann im Falle einer RSA das Ende des freien Sehnenanteils ebenfalls als „Füllmaterial“ im Sinne einer Interpositions-Arthroplastik eingelegt werden.

\subsubsection{Nachbehandlung}

Abhängig vom gewählten Operationsverfahren variiert auch die Nachbehandlung. Im Allgemeinen finden zur Verringerung der postoperativen Ödembildung und Schmerzen sowie zur Verbesserung der Wundheilung eine adäquate Analgesie, eine Kryotherapie bei Bedarf sowie die Ruhigstellung des Daumens in Hochlagerung Anwendung. Der Daumen wird bei den Resektionssuspensionsarthroplastiken (je nach Autor) mittels palmarer Gipsschiene mit Daumeneinschluss oder Abduktionswatteverbands für drei bis sechs Wochen ruhiggestellt (PECHLANER et al., 1998). Hierdurch soll genug Zeit gewährleistet werden, damit die Sehnenrespektive Bandplastik suffizient einheilen kann. Im Anschluss daran erfolgt zunächst die belastungsfreie, später eine schrittweise belastungsintensivere Beübung des Daumens, durch die sukzessive sowohl die Opposition als auch die Retroversion des Daumens aktiv und passiv verbessert werden sollen. Bis zur kompletten Rehabilitation und vollen Belastbarkeit des Daumens können nach der OP 8-12 Wochen vergehen. Durch regelmäßiges Training der atrophierten Muskulatur können auch noch innerhalb des ersten Jahres Verbesserungen eintreten. Physio- und ergotherapeutische Mitbehandlungen können hilfreich sein, den Prozess zu beschleunigen und das oben genannte Rehabilitationsziel zu erreichen (RICHTER, 2015). Sofern keine Komplikationen vorliegen, erfordert eine mitbehandelte STTArthrose keine zusätzliche Nachbehandlung.

$\mathrm{Ab}$ der 5. postoperativen Woche können bei Verwachsungen und Narbenbildungen im Thenarbereich spezielle Narbenmassagen und Ultraschall angewendet werden. Insbesondere die gelegentlich auftretenden, durch Narben induzierten Hyper- und Parästhesien des sensiblen Astes des $\mathrm{N}$. radialis werden durch desensibilisierende Massagen und Maßnahmen behandelt (ISEL und CÉLÉRIER, 2009). 


\subsection{Material und Methoden der Nachuntersuchung}

Die Nachuntersuchungen zur Datenerhebung für die vorliegende Arbeit fanden im Zeitraum zwischen März und August 2018 statt und der postoperative Zeitraum betrug mindestens 12 Monate. An den Untersuchungsterminen wurde zuerst mittels Fragebogen das Geschlecht, das Alter, die manuelle Dominanz sowie der TDX-Score und die subjektive Lebensqualitätseinschätzung ermittelt. Daraufhin wurden in einem Gespräch Rückfragen der Studienteilnehmer geklärt und im Anschluss die klinische Untersuchung durchgeführt. Die Oppositionsfähigkeit des Daumens wurde mittels Kapandji-Score und die Kraft im Grobgriff, Schlüsselgriff, palmaren Spitzgriff und Dreifingerspitzgriff mittels Martin-Vigorimeter gemessen. Zudem wurde die Schmerzintensität in Ruhe sowie bei leichter und intensiver Belastung mittels visueller Analogskala bestimmt. Im Folgenden werden die genutzten Techniken und Instrumente kurz vorgestellt.

\subsubsection{Thumb Disability Examination}

Die Thumb Disability Examination (TDX) ist ein Instrument, um das Ergebnis nach Rhizarthrose-Therapie zu bewerten. Der Fragebogen beinhaltet 20 Fragen und ist in drei Kategorien aufgeteilt. Die erste Kategorie erfasst mit 11 Fragen das Ausmaß der Schwierigkeiten bei Bewältigung alltäglicher Aktivitäten, die mit der betroffenen Hand (z. B. einen Schlüssel umdrehen, schreiben, ein Glas Wasser halten, etc.) bzw. mit beiden Händen (z. B. Schuhe zubinden, Wäsche auswringen, etc.) ausgeführt werden. In der zweiten Kategorie werden mit 5 Fragen die Ausprägung der Symptome sowie die Auswirkung auf die Stimmung erfasst. In der letzten Kategorie werden die subjektive Einschätzung und Zufriedenheit bezüglich der Bewegungsfähigkeit, der Stärke, des Schmerzlevels und der Gesamtfunktionalität der betroffenen Hand abgefragt. Für jede Frage steht eine 1-5 Punkteskala zur Verfügung, wobei 1 Punkt das bestmögliche und 5 Punkte das schlechteste Ergebnis darstellen. Der Test kann als Ganzes, die Kategorien jedoch auch einzeln berechnet und bewertet werden. Die Wertung findet durch eine Skala von 0 bis 100 Punkten statt, wobei 0 Punkte den geringsten Wert des Behinderungsgrads und 100 Punkte den höchsten darstellen. Die Gleichung zur Berechnung der einzelnen Scores ist unten aufgeführt. Es dürfen nicht mehr als $10 \%$ der Fragen unausgefüllt sein, da der TDX-Score sonst nicht errechnet werden kann (NOBACK et al., 2016).

$$
\begin{aligned}
& \text { Score }=\left(\frac{N}{Q-B}-1\right) * 25 \\
& N=\text { Summe der beantworteten Fragen im gesamten TDX oder den einzelnen Kategorien } \\
& Q=\text { Für den gesamten TDX: 20, für die einzelnen Kategorien: die Anzahl der dort gestellten Fragen } \\
& B=\text { Anzahl der unbeantworteten Fragen }
\end{aligned}
$$




\subsubsection{Visuelle Analogskala (VAS)}

Die visuelle Analogskala findet häufig Anwendung, um Schmerzen oder das subjektive Empfinden von Patienten einzuschätzen. Hierbei setzt der Befragte eine Markierung entsprechend der Empfindung auf einer Linie ohne Skalierung, deren linker Endpunkt „keine Schmerzen“ und rechter Endpunkt „unerträgliche Schmerzen“ bedeutet. Im Anschluss daran wird die Markierung durch den Untersucher mittels definierter Skala ( $0=$ keine Schmerzen, 10 = unerträgliche Schmerzen) abgelesen und dokumentiert (BIJUR et al., 2001).

\subsubsection{Funktionelle Klassifikation nach KAPANDJI / Kapandji-Score}

Mit der funktionellen Klassifikation nach KAPANDJ, auch Kapandji-Score genannt, kann der Status respektive der funktionelle Fortschritt der Patienten objektiv nachvollzogen werden. Hierbei wird mittels 10-Punkte-System die kombinierte Funktionalität des Daumensattelgelenks, Daumengrundgelenks und Interphalangealgelenks insbesondere im Hinblick auf die Opposition bewertet. Die Bewertung erfolgt entsprechend der Punkte, die der Patient mit dem Daumen berühren kann. Die Punkte können der unten aufgeführten Tabelle entnommen werden (KAPANDJI, 1986).

\begin{tabular}{|l|l|}
\hline Score & Berührungspunkte \\
\hline 1 & Radialseite der Grundphalanx des Zeigefingers \\
\hline 2 & Radialseite der Mittelphalanx des Zeigefingers \\
\hline 3 & Spitze des Zeigefingers \\
\hline 4 & Spitze des Mittelfingers \\
\hline 5 & Spitze des Ringfingers \\
\hline 6 & Spitze des Kleinfingers \\
\hline 7 & Beugefalte des Kleinfingers am DIP-Gelenk \\
\hline 8 & Beugefalte des Kleinfingers am PIP-Gelenk \\
\hline 9 & Beugefalte des Kleinfingers am Grundgelenk \\
\hline 10 & Distale Beugefalte der Handinnenfläche \\
\hline
\end{tabular}

Tabelle Kapandji-Score (KAPANDJI, 1986).

\subsubsection{Kraftmessung mittels Martin-Vigorimeter}

Die Messung der maximalen Kraft in verschiedenen Griffen wurde mit Hilfe des Martin-Vigorimeters ermittelt. Es wurden Druckbälle in verschiedenen Größen verwendet, um dem Patienten beim Zusammendrücken die Entfaltung der Maximalkraft zu ermöglichen. Für Patienten mit einem Mittelhandumfang von mehr als $19 \mathrm{~cm}$ wurde im Grobgriff der Druckball mit einem Durchmesser von $6 \mathrm{~cm}$ verwendet. Bei einem Mittelhandumfang von weniger als 19 $\mathrm{cm}$ fand der mittlere Ball mit einem Durchmesser von 4,5 cm Anwendung. Für die Messungen im Schlüsselgriff, palmaren Spitzgriff sowie im Dreifingerspitzgriff wurde der kleine Ball 
mit einem Durchmesser von $4 \mathrm{~cm}$ genutzt. Die Untersuchung wurde am Tisch sitzend mit im Ellenbogengelenk 90 Grad flektiertem Arm und auf dem Tisch ruhendem Unterarm durchgeführt. Die Messungen wurden für jeden Griff jeweils drei Mal auf der betroffenen sowie auf der gesunden Seite mit einer Pause von 30 Sekunden zwischen den einzelnen Durchgängen vorgenommen. Aus den jeweils drei bestimmten Werten der einzelnen Griffe wurde im Anschluss der Mittelwert berechnet und dokumentiert (DESROSIERS et al., 1995; SIPERS et al., 2016). 


\section{Literaturverzeichnis}

AbZUG, J.M., Osterman, A.L., 2011. Arthroscopic hemiresection for stage II-III trapeziometacarpal osteoarthritis. Hand Clin. 27, 347-354. https://doi.org/10.1016/j.hcl.2011.05.009

Armstrong, A.L., HUnTER, J.B., DAVIS, T.R.C., 1994. The prevalence of degenerative arthritis of the base of the thumb in post-menopausal women. J. Hand Surg. Am. 19, 340-341. https://doi.org/10.1016/0266-7681(94)90085-X

ATESHIAN, G.A., RosenwASSER, M.P., Mow, V.C., 1992. Curvature characteristics and congruence of the thumb carpometacarpal joint: Differences between female and male joints. J. Biomech. 25, 591-607. https://doi.org/10.1016/0021-9290(92)90102-7

BADIA, A., 2007. Arthroscopy of the trapeziometacarpal and metacarpophalangeal joints. J. Hand Surg. Am. 32, 707-724. https://doi.org/10.1016/j.jhsa.2007.02.020

BaKeR, R.H.J., Al-ShukRI, J., DAVIS, T.R.C., 2017. Evidence-Based Medicine: Thumb Basal Joint Arthritis. Plast. Reconstr. Surg. 139, 256e-266e. https://doi.org/10.1097/PRS.0000000000002858

Barrera-Ochoa, S., Vidal-Tarrason, N., Correa-Vázquez, E., ReVerte-Vinaixa, M.M., FontSegura, J., Mir-Bullo, X., 2014. Pyrocarbon interposition (PyroDisk) implant for trapeziometacarpal osteoarthritis: minimum 5-year follow-up. J. Hand Surg. Am. 39, 2150-2160. https://doi.org/10.1016/j.jhsa.2014.07.011

BERGER, R.A., 1997. A technique for arthroscopic evaluation of the first carpometacarpal joint. J. Hand Surg. Am. 22, 1077-1080. https://doi.org/10.1016/S0363-5023(97)80052-4

Bettinger, P.C., Berger, R.A., 2001. Functional ligamentous anatomy of the trapezium and trapeziometacarpal joint (gross and arthroscopic). Hand Clin. 17, 151-68, vii.

Bettinger, P.C., LinsCheid, R.L., Berger, R.A., CoOney, W.P., AN, K.-N., 1999. An anatomic study of the stabilizing ligaments of the trapezium and trapeziometacarpal joint. J. Hand Surg. Am. 24, 786-798. https://doi.org/10.1053/jhsu.1999.0786

BetTINGER, P.C., SMUTZ, W.P., LINSCHEID, R.L., COONEY, W.P., AN, K.N., 2000. Material properties of the and trapeziometacarpal ligaments. J. Hand Surg. Am. https://doi.org/10.1053/jhsu.2000.18487

BiJur, P., Silver, W., Gallagher, E., 2001. Reliability of the visual analog scale for measurement of acute pain. Acad. Emerg. Med. https://doi.org/10.1111/j.1553-2712.2001.tb01132.x

Botha-Scheepers, S., Riyazi, N., Watt, I., Rosendaal, F.R., Slagboom, E., Bellamy, N., Breedveld, F.C., KLopPenBuRG, M., 2009. Progression of hand osteoarthritis over 2 years: a clinical and radiological follow-up study. Ann. Rheum. Dis. 68, 1260-1264. https://doi.org/10.1136/ard.2008.087981

Braun, B., Veith, N., Frueh, F., Klein, M., Knopp, W., Pohlemann, T., 2016. Die Rettungsoperation mittels Mini TightRope ${ }^{\circledR}$ bei schmerzhafter Proximalisierung des 1. Mittelhandknochens nach Trapezektomie. Handchir Mikrochir Plast Chir. 48, 300-305. https://doi.org/10.1055/s-0042113774

BrunelLi, G., MoninI, L., BrunelLI, F., 1989. Stabilisation of the trapezio-metacarpal joint. J. Hand Surg. J. Br. Soc. Surg. Hand 14, 209-212. https://doi.org/10.1016/0266-7681(89)90128-9

Buhler, M., Chapple, C.M., Stebbings, S., Sangelajl, B., Baxter, G.D., 2019. Effectiveness of splinting for pain and function in people with thumb carpometacarpal osteoarthritis: a systematic review with meta-analysis. Osteoarthr. Cartil. 27, 547-559. https://doi.org/10.1016/j.joca.2018.09.012

Casimiro, L., Brosseau, L., Welch, V., Milne, S., Judd, M., Wells, G.A., Tugwell, P., Shea, B., 2002. Therapeutic ultrasound for the treatment of rheumatoid arthritis. Cochrane Database Syst. Rev. https://doi.org/10.1002/14651858.CD003787

Cebrian-Gomez, R., Lizaur-Utrilla, A., Sebastia-Forcada, E., Lopez-Prats, F.A., 2019. Outcomes 
of cementless joint prosthesis versus tendon interposition for trapeziometacarpal osteoarthritis: a prospective study. J. Hand Surg. Eur. Vol. 44, 151-158. https://doi.org/10.1177/1753193418787151

Cheema, T., Salas, C., Morrell, N., Lansing, L., Reda Taha, M.M., Mercer, D., 2012. Opening wedge trapezial osteotomy as possible treatment for early trapeziometacarpal osteoarthritis: a biomechanical investigation of radial subluxation, contact area, and contact pressure. J. Hand Surg. Am. 37, 699-705. https://doi.org/10.1016/j.jhsa.2012.01.013

ChUG, M., Williams, N., BENN, D., BRINDLEY, S., 2014. Outcome of uncemented trapeziometacarpal prosthesis for treatment of thumb carpometacarpal joint arthritis. Indian J. Orthop. 48, 394. https://doi.org/10.4103/0019-5413.136270

CobB, T., SteRBANK, P., LEMKE, J., 2011. Arthroscopic resection arthroplasty for treatment of combined carpometacarpal and scaphotrapeziotrapezoid (pantrapezial) arthritis. J. Hand Surg. Am. 36, 413-419. https://doi.org/10.1016/j.jhsa.2010.11.039

COLDITZ, J.C., 2000. The biomechanics of a thumb carpometacarpal immobilization splint: Design and fitting. J. Hand Ther. 13, 228-235. https://doi.org/10.1016/S0894-1130(00)80006-X

Colman, M., Mass, D.P., Draganich, L.F., 2007. Effects of the deep anterior oblique and dorsoradial ligaments on trapeziometacarpal joint stability. J. Hand Surg. Am. 32, 310-317. https://doi.org/10.1016/j.jhsa.2006.12.002

COONEY, W., ChAO, E., 1977. Biomechanical analysis of static forces in the thumb during hand function. J. Bone Jt. Surg. 59, 27-36. https://doi.org/10.2106/00004623-197759010-00004

COONEY, W.P., LEDDY, T.P., LARSON, D.R., 2006. Revision of Thumb Trapeziometacarpal Arthroplasty. J. Hand Surg. Am. 31, 219.e1-219.e9. https://doi.org/10.1016/j.jhsa.2005.10.018

Dahaghin, S., Bierma-Zeinstra, S.M.A., Ginal, A.Z., Pols, H.A.P., Hazes, J.M.W., Koes, B.W., 2005. Prevalence and pattern of radiographic hand osteoarthritis and association with pain and disability (the Rotterdam study). Ann. Rheum. Dis. 64, 682-687. https://doi.org/10.1136/ard.2004.023564

DavenPort, B.J., Jansen, V., Yeandle, N., 2012. Pilot randomized controlled trial comparing specific dynamic stability exercises with general exercises for thumb carpometacarpal joint osteoarthritis. Hand Ther. 17, 60-67. https://doi.org/10.1258/ht.2012.012010

Day, C.S., Gelberman, R., Patel, A.A., Vogt, M.T., Ditsios, K., Boyer, M.I., 2004. Basal joint osteoarthritis of the thumb: a prospective trial of steroid injection and splinting. J. Hand Surg. Am. 29, 247-251. https://doi.org/10.1016/j.jhsa.2003.12.002

DEILER, S., 2019. Daumensattelgelenkarthrose. Orthopade 48, 351-366. https://doi.org/10.1007/s00132-019-03707-8

Desrosiers, J., Hébert, R., Bravo, G., Dutil, E., 1995. Comparison of the Jamar dynamometer and the Martin vigorimeter for grip strength measurements in a healthy elderly population. Scand. J. Rehabil. Med. 27, 137-43.

Drewniany, J.J., Palmer, A.K., Flatt, A.E., 1985. The scaphotrapezial ligament complex: An anatomic and biomechanical study. J. Hand Surg. Am. 10, 492-498. https://doi.org/10.1016/S0363-5023(85)80070-8

Eaton, R.G., Glickel, S.Z., 1987. Trapeziometacarpal osteoarthritis. Staging as a rationale for treatment. Hand Clin. 3, 455-71.

EATON, R.G., LANE, L.B., LiTTLER, J.W., KeYSER, J.J., 1984. Ligament reconstruction for the painful thumb carpometacarpal joint: A long-term assessment. J. Hand Surg. Am. 9, 692-699. https://doi.org/10.1016/S0363-5023(84)80015-5

EATON, R.G., LITTLER, J.W., 1973. Ligament reconstruction for the painful thumb carpometacarpal joint. J. Bone Joint Surg. Am. 55, 1655-66. https://doi.org/10.2106/00004623-197355080-00010

EBENBICHLER, G., 2009. Evidenzbasierte Medizin und Ultraschalltherapie des Skeletts. Z. Rheumatol. 68, 543-548. https://doi.org/10.1007/s00393-009-0457-z 
EDMUNDS, J.O., 2011. Current concepts of the anatomy of the thumb trapeziometacarpal joint. J. Hand Surg. Am. 36, 170-182. https://doi.org/10.1016/j.jhsa.2010.10.029

EdWARDS, S.G., Ramsey, P.N., 2010. Prospective outcomes of stage III thumb carpometacarpal arthritis treated with arthroscopic hemitrapeziectomy and thermal capsular modification without interposition. J. Hand Surg. Am. 35, 566-571. https://doi.org/10.1016/j.jhsa.2009.12.022

EPPING, W., 1989. Die Suspensionsplastik zur Behandlung der Sattelgelenksarthrose. Oper. Orthop. Traumatol. 1, 100-108. https://doi.org/10.1007/BF02581211

Fassbender, H.G., 1984. Rheumatoide Arthritis, in: DoerR, W., Seifert, G., Uehlinger, E. (Hrsg.), Pathologie der Gelenke und Weichteiltumoren, Band 18 von Spezielle pathologische Anatomie. Springer, Berlin/Heidelberg/New York/Tokyo, S. 191-229.

Florack, T.M., Miller, R.J., Pellegrini, V.D., Burton, R.I., Dunn, M.G., 1992. The prevalence of carpal tunnel syndrome in patients with basal joint arthritis of the thumb. J. Hand Surg. Am. 17, 624-630. https://doi.org/10.1016/0363-5023(92)90305-9

Fontana, L., Neel, S., Claise, J.-M., Ughetto, S., Catilina, P., 2007. Osteoarthritis of the thumb carpometacarpal joint in women and occupational risk factors: a case-control study. J. Hand Surg. Am. 32, 459-65. https://doi.org/10.1016/j.jhsa.2007.01.014

FORESTIER, J., 1937. L'ostéoarthrite sèche trapézo-métacarpienne (rhizarthrose du pouce). Press. Med 315-317.

Foucher, G., Pretz, P.L., ERHARD, L., 1998. La dénervation articulaire, une réponse simple à des problèmes complexes de chirurgie de la main. Chirurgie 123, 183-188. https://doi.org/10.1016/S0001-4001(98)80104-9

Fowler, A., Swindells, M.G., BuRKE, F.D., 2015. Intra-articular corticosteroid injections to manage trapeziometacarpal osteoarthritis-a systematic review. Hand (N. Y). 10, 583-92. https://doi.org/10.1007/s11552-015-9778-3

Frank, U., 2015. Die Untersuchung des Handgelenks, in: Sauerbier, M., Eisenschenk, A., Krimmer, H., PARTECKE, B.-D., SchalleR, H.-E. (Hrsg.), Die Handchirurgie. Elsevier GmbH, München, S. 18-26.

Gabriel, M., Poeppel, T.D., Farahatı, J., Krause, T., Buchmann, I., Panholzer, P., Puchner, R., Fischer, M., Mödder, G., Becherer, A., Kampen, W., Kurth, J., Krause, B.J., Wissmeyer, M., KRESNIK, E., 2019. Gemeinsame Handlungsempfehlung (S1-Leitlinie) der DGN, OGNMB, SGNM Radiosynoviorthese (RSO) URL: https://www.nuklearmedizin.de/leistungen/leitlinien/docs/031 023_9-2019.pdf (zugegriffen am 04.06.2020).

Gander, B., Wollstein, R., 2013. Surgical Treatment for Thumb CMC Joint Arthritis. Curr. Rheumatol. Rev. 9, 113-8. https://doi.org/10.2174/1573397111309020006

GeRVIS, W.H., 1948. Excision of the trapezium for osteoarthritis of the trapezio-metacarpal joint. Postgrad. Med. J. 24, 262-4. https://doi.org/10.1136/pgmj.24.271.262

GLICKEL, S.Z., 2001. Clinical assessment of the thumb trapeziometacarpal joint. Hand Clin. 17, 18595.

Grifka, J.K., Zacher, J., Brown, J.P., Seriolo, B., Lee, A., Moore, A., Gimona, A., 2004. Efficacy and tolerability of lumiracoxib versus placebo in patients with osteoarthritis of the hand. Clin. Exp. Rheumatol. 22, 589-596.

Haugen, I.K., Englund, M., Aliabadi, P., Niu, J., Clancy, M., Kvien, T.K., Felson, D.T., 2011. Prevalence, incidence and progression of hand osteoarthritis in the general population: the Framingham Osteoarthritis Study. Ann. Rheum. Dis. 70, 1581-1586. https://doi.org/10.1136/ard.2011.150078

Horch, R.E., Unglaub, F., 2011. Rhizarthrose, in: Towfigh, H., Hierner, R., Langer, M., R, F. (Hrsg.), Handchirurgie. Springer, Berlin, Heidelberg, S. 1401-1411. https://doi.org/10.1007/978-3-64211758-9_51

IMAEDA, T., AN, K.-N., COONEY, W.P., LINSCHEID, R., 1993. Anatomy of trapeziometacarpal ligaments. 


\section{J. Hand Surg. Am. 18, 226-231. https://doi.org/10.1016/0363-5023(93)90352-4}

ISEL, M., CÉlÉRIER, S., 2009. Rhizarthrose: Nachbehandlung und Orthesen, in: MERLE, M., REHART, S. (Hrsg.), Chirurgie der Hand: Rheuma, Arthrose, Nervenengpässe. Georg Thieme Verlag KG, Stuttgart/New York, S. 56-58.

Jensen, V., BøgGILD, H., Johansen, J.P., 1999. Occupational use of precision grip and forceful gripping, and arthrosis of finger joints: A literature review. Occup. Med. (Chic. III). 49, 383-388. https://doi.org/10.1093/occmed/49.6.383

Kaltenborn, A., Bulling, E., Nitsche, M., Carl, U.M., Hermann, R.M., 2016. The field size matters: low dose external beam radiotherapy for thumb carpometacarpal osteoarthritis. Strahlentherapie und Onkol. 192, 582-588. https://doi.org/10.1007/s00066-016-0995-7

KAPANDJI, A., 1986. Cotation clinique de l'opposition et de la contre-opposition du pouce - Clinical opposition and reposition test of the thumb. Ann. Chir. Main 5, 67-73. https://doi.org/10.1016/s0753-9053(86)80053-9

Katzel, E.B., Bielicka, D., ShakiR, S., Fowler, J., Buterbaugh, G.A., Imbriglia, J.E., 2016. Midcarpal and Scaphotrapeziotrapezoid Arthritis in Patients with Carpometacarpal Arthritis. Plast. Reconstr. Surg. 137, 1793-1798. https://doi.org/10.1097/PRS.0000000000002160

Kroon, F.P.B., Rubio, R., Schoones, J.W., Kloppenburg, M., 2016. Intra-Articular Therapies in the Treatment of Hand Osteoarthritis: A Systematic Literature Review. Drugs Aging 33, 119-33. https://doi.org/10.1007/s40266-015-0330-5

Kwok, W.Y., Kloppenburg, M., Marshall, M., Nicholls, E., Rosendaal, F.R., Peat, G., 2014. The prevalence of erosive osteoarthritis in carpometacarpal joints and its clinical burden in symptomatic community-dwelling adults. Osteoarthr. Cartil. 22, 756-63. https://doi.org/10.1016/j.joca.2014.03.012

LORÉA, P.D., 2003. First carpometacarpal joint denervation: anatomy and surgical technique. Tech. Hand Up. Extrem. Surg. 7, 26-31. https://doi.org/10.1097/00130911-200303000-00006

Maddali-Bongi, S., Del Rosso, A., Galluccio, F., Sigismondi, F., Matucci-Cerinic, M., 2016. Is an intervention with a custom-made splint and an educational program useful on pain in patients with trapeziometacarpal joint osteoarthritis in a daily clinical setting? Int. J. Rheum. Dis. 19, 773780. https://doi.org/10.1111/1756-185X.12318

Mariconda, M., Russo, S., Smeraglia, F., Busco, G., 2014. Partial trapeziectomy and pyrocarbon interpositional arthroplasty for trapeziometacarpal joint osteoarthritis: results after minimum 2 years of follow-up. J. Hand Surg. Eur. Vol. 39, 604-610. https://doi.org/10.1177/1753193413519384

Meier, R., Frey, S., Kenn, W., Schmitt, S., Meffert, R., 2011. Prävalenz der Rhizarthrose. Obere Extrem. 6, 115-117. https://doi.org/10.1007/s11678-011-0119-z

MENON, J., 1996. Arthroscopic management of trapeziometacarpal joint arthritis of the thumb. Arthrosc. J. Arthrosc. Relat. Surg. 12, 581-587. https://doi.org/10.1016/S0749-8063(96)90198$\mathrm{X}$

Merle, M., Voche, P., 2009. Rhizarthrose, in: Merle, M., Rehart, S. (Hrsg.), Chirurgie der Hand: Rheuma, Arthrose, Nervenengpässe. Georg Thieme Verlag KG, Stuttgart/New York, S. 25-58.

Merritt, M.M., Roddey, T.S., Costello, C., Olson, S., 2010. Diagnostic value of clinical grind test for carpometacarpal osteoarthritis of the thumb. J. Hand Ther. 23, 261-7; quiz 268. https://doi.org/10.1016/j.jht.2010.02.001

Miura, T., OHe, T., Masuko, T., 2004. Comparative in vivo kinematic analysis of normal and osteoarthritic trapeziometacarpal joints. J. Hand Surg. Am. 29, 252-257. https://doi.org/10.1016/j.jhsa.2003.11.002

Nanno, M., Kodera, N., Tomori, Y., Hagiwara, Y., TAKal, S., 2017. Three-dimensional dynamic motion analysis of the first carpometacarpal ligaments. J. Orthop. Surg. 25, 230949901668475. https://doi.org/10.1177/2309499016684752 
Noback, P., Lombardi, J., Seetharaman, M., Lee, D., Strauch, R., Rosenwasser, M., 2016. Development and Validation of a Disease-Specific Questionnaire for Basal Joint Arthritis. J. Wrist Surg. 6, 126-133. https://doi.org/10.1055/s-0036-1593612

NoRTH, E.R., RUtLEDGE, W.M., 1983. The trapezium-thumb metacarpal joint: the relationship of joint shape and degenerative joint disease. Hand 15, 201-6. https://doi.org/10.1016/s0072968x(83)80014-x

Østerås, N., KJeken, I., Smedslund, G., Moe, R.H., Slatkowsky-Christensen, B., Uhlig, T., Hagen, K.B., 2017. Exercise for hand osteoarthritis: A cochrane systematic review. J. Rheumatol. 44, 1850-1858. https://doi.org/10.3899/jrheum.170424

PARKER, W.L., LINSCHEID, R.L., AMADIO, P.C., 2008. Long-term outcomes of first metacarpal extension osteotomy in the treatment of carpal-metacarpal osteoarthritis. J. Hand Surg. Am. 33, 1737-43. https://doi.org/10.1016/j.jhsa.2008.08.003

Pechlaner, S., Hussl, H., Kerschbaumer, F., Poisel, S., Gratzer, J., 1998. Operationsatlas Handchiurgie, 1. ed. Georg Thieme Verlag, Stuttgart/New York.

PELLEGRINI, V.D., 2001. Pathomechanics of the thumb trapeziometacarpal joint. Hand Clin. 17, 17584 , vii-viii.

Pellegrini, V.D., Parentis, M., Judkins, A., Olmstead, J., Olcott, C., 1996. Extension metacarpal osteotomy in the treatment of trapeziometacarpal osteoarthritis: A biomechanical study. J. Hand Surg. Am. 21, 16-23. https://doi.org/10.1016/S0363-5023(96)80149-3

Pieron, A.P., 1973. The mechanism of the first carpometacarpal (CMC) joint. An anatomical and mechanical analysis. Acta Orthop. Scand. Suppl. 148, 1-104. https://doi.org/10.3109/ort.1973.44.suppl-148.01

Raven, E.E.J., Kerkhoffs, G.M.M.J., Rutten, S., Marsman, A.J.W., Marti, R.K., Albers, G.H.R., 2007. Long term results of surgical intervention for osteoarthritis of the trapeziometacarpal joint. Int. Orthop. 31, 547-554. https://doi.org/10.1007/s00264-006-0217-5

Richter, M., 2015. Rhizarthrose, in: Sauerbier, M., Eisenschenk, A., Krimmer, H., Partecke, B.-D., SchalleR, H.-E. (Hrsg.), Die Handchirurgie. Elsevier GmbH, München, S. 107-120.

Riyazi, N., Rosendaal, F.R., Slagboom, E., Kroon, H.M., Breedveld, F.C., Kloppendurg, M., 2008. Risk factors in familial osteoarthritis: the GARP sibling study. Osteoarthr. Cartil. 16, 654-659. https://doi.org/10.1016/j.joca.2007.10.012

Rust, P.A., ThAM, S.K.Y., 2011. Ligament reconstruction of the Trapezial-metacarpal joint for early arthritis: a preliminary report. J. Hand Surg. Am. 36, 1748-52. https://doi.org/10.1016/j.jhsa.2011.07.026

Schibli-Beer, S., Mark, G., Canova, M., 2008. Ergebnisse nach alleiniger Trapezektomie und Kapselraffung bei der Behandlung der Rhizarthrose. Handchir Mikrochir Plast Chir. 40, 169-174. https://doi.org/10.1055/s-2007-989389

SCHMIDT, H.-M., LANZ, U., 2013a. Daumensattelgelenk, in: SCHMIDT, H.-M., LANZ, U. (Hrsg.), Chirurgische Anatomie der Hand. Georg Thieme Verlag, Stuttgart, S. 106-109. https://doi.org/10.1055/b-002-13404

SchmidT, H.-M., LANZ, U., 2013b. Skelett der Handwurzel, in: ScHMIDT, H.-M., LANZ, U. (Hrsg.), Chirurgische Anatomie der Hand. Georg Thieme Verlag, Stuttgart, S. 44-52. https://doi.org/10.1055/b-002-13404

SCHMITT, R., HAHN, P., 2014. Anatomie der Mittelhand und Finger, in: ScHMITT, R., LANZ, U. (Hrsg.), Bildgebende Diagnostik der Hand. Georg Thieme Verlag KG, Stuttgart, S. 152-183.

SchmitT, R., UnGLAUB, F., 2014. Anatomie des Unterarms und der Handwurzel, in: ScHMITT, R., LANZ, U. (Hrsg.), Bildgebende Diagnostik der Hand. Georg Thieme Verlag KG, Stuttgart, S. 108-151.

Sigfusson, R., LUNDBORG, G., 1991. Abductor pollicis longus tendon arthroplasty for treatment of arthrosis in the first carpometacarpal joint. Scand. J. Plast. Reconstr. Surg. hand Surg. 25, $73-$ 7. https://doi.org/10.3109/02844319109034926 
SiPERS, W.M.W.H., VerdiJK, L.B., SiPERS, S.J.E., SchOlS, J.M.G.A., VAN LOON, L.J.C., 2016. The Martin Vigorimeter Represents a Reliable and More Practical Tool Than the Jamar Dynamometer to Assess Handgrip Strength in the Geriatric Patient. J. Am. Med. Dir. Assoc. 17, 466.e1-7. https://doi.org/10.1016/j.jamda.2016.02.026

StÄBleR, A., WAGNeR, M., 2014. Arthrosis deformans, in: Schmitt, R., LANZ, U. (Hrsg.), Bildgebende Diagnostik der Hand. Georg Thieme Verlag KG, Stuttgart, S. 392-407.

SWANSON, A.B., 1972. Disabling arthritis at the base of the thumb: treatment by resection of the trapezium and flexible (silicone) implant arthroplasty. J. Bone Joint Surg. Am. 54, 456-71. https://doi.org/10.2106/00004623-197254030-00002

SWIGART, C.R., 2008. Arthritis of the base of the thumb. Curr. Rev. Musculoskelet. Med. 1, 142-146. https://doi.org/10.1007/s12178-008-9022-7

SWIGART, C.R., EATON, R.G., GLICKEL, S.Z., JOHNSON, C., 1999. Splinting in the treatment of arthritis of the first carpometacarpal joint. J. Hand Surg. Am. 24, 86-91. https://doi.org/10.1053/jhsu.1999.jhsu24a0086

Szalay, G., Meyer, C., Kraus, R., Heiss, C., Schnettler, R., 2009. Die operative Versorgung der Rhizarthrose mittels Pyrocarbonspacer als Trapeziumersatz. Handchir Mikrochir Plast Chir. 41, 300-305. https://doi.org/10.1055/s-0029-1238281

Szalay, G., Scheufens, T., Alt, V., Boecker, W., Schnettler, R., 2014. Die operative Versorgung der therapierefraktären Rhizarthrose mittels Trapezektomie und Suspension des ersten Strahls mittels Mini TightRope ${ }^{\circledR}$. Handchir Mikrochir Plast Chir. 46, 179-185. https://doi.org/10.1055/s0034-1368715

Szalay, G., Scheufens, T., Alt, V., Thormann, U., Heiss, C., 2015. Erfahrungen mit dem Mini TightRope $\AA$ bei schmerzhafter Proximalisierung des 1. Mittelhandstrahls nach Trapezektomie bei Rhizarthrose. Handchir Mikrochir Plast Chir. 47, 17-23. https://doi.org/10.1055/s-00341398608

Szalay, G., Schleicher, I., Alt, V., Pavlidis, T., Schnettler, R., 2011. Operative Versorgung der Rhizarthrose. Orthopade 40, 237-246. https://doi.org/10.1007/s00132-010-1729-7

TaN, J., Xu, J., XIE, R.G., Deng, A.D., TANG, J.B., 2011. In vivo length and changes of ligaments stabilizing the thumb carpometacarpal joint. J. Hand Surg. Am. 36, 420-7. https://doi.org/10.1016/j.jhsa.2010.11.007

TAYLOR, E.J., DESARI, K., D'ARCY, J.C., BONNICI, A. V, 2005. A comparison of fusion, trapeziectomy and silastic replacement for the treatment of osteoarthritis of the trapeziometacarpal joint. $\mathrm{J}$. Hand Surg. Br. 30, 45-9. https://doi.org/10.1016/j.jhsb.2004.08.006

Tomaino, M.M., 2011. Basal metacarpal osteotomy for osteoarthritis of the thumb. J. Hand Surg. Am. 36, 1076-9. https://doi.org/10.1016/j.jhsa.2011.03.035

Vermeulen, G.M., Brink, S.M., Slijper, H., Feitz, R., Moojen, T.M., Hovius, S.E.R., Selles, R.W., 2014. Trapeziometacarpal arthrodesis or trapeziectomy with ligament reconstruction in primary trapeziometacarpal osteoarthritis: a randomized controlled trial. J. Bone Joint Surg. Am. 96, 72633. https://doi.org/10.2106/JBJS.L.01344

Vermeulen, G.M., Slijper, H., Feitz, R., Hovius, S.E.R., Moojen, T.M., Selles, R.W., 2011. Surgical management of primary thumb carpometacarpal osteoarthritis: a systematic review. J. Hand Surg. Am. 36, 157-69. https://doi.org/10.1016/j.jhsa.2010.10.028

Villafañe, J.H., Silva, G.B., Fernandez-CARnero, J., 2012. Effect of thumb joint mobilization on pressure pain threshold in elderly patients with thumb carpometacarpal osteoarthritis. J. Manipulative Physiol. Ther. 35, 110-20. https://doi.org/10.1016/j.jmpt.2011.12.002

WAGENHÄUSER, F., 1969. Die Rheumamorbidität. Eine klinisch-epidemiologische Untersuchung. Hans Huber, Bern.

WAJON, A., AdA, L., EDMUNDS, I., 2005. Surgery for thumb (trapeziometacarpal joint) osteoarthritis. Cochrane database Syst. Rev. CD004631. https://doi.org/10.1002/14651858.CD004631.pub2 
Warganich, T., Shin, A.Y., 2017. A Technique for the Management of Concomitant Scaphotrapezoid Arthritis in Patients With Thumb Metacarpotrapezial Arthritis: Interposition Arthroplasty With a Capitate Suture Anchor. Tech. Hand Up. Extrem. Surg. 21, 71-74. https://doi.org/10.1097/BTH.0000000000000155

WATSON, H.K., BALLET, F.L., 1984. The SLAC wrist: scapholunate advanced collapse pattern of degenerative arthritis. J. Hand Surg. Am. 9, 358-65. https://doi.org/10.1016/s03635023(84)80223-3

WILDER, F. V, BARRETT, J.P., FARINA, E.J., 2006. Joint-specific prevalence of osteoarthritis of the hand. Osteoarthr. Cartil. 14, 953-7. https://doi.org/10.1016/j.joca.2006.04.013

WILHELM, A., 1972. Die Eingriffe zur Schmerzausschaltung durch Denervation. In: WACHSMUTH, W., Wilhelm, A. (Hrsg.), Die Operation an der Hand. In: Zenker, R., Heberer, G., HegemanN, G. (Hrsg.): Allgemeine und Spezielle Operationslehre. Band10/3. Springer, Berlin/Heidelberg/New York.

WiLHELM, A., 1966. Die Gelenkdenervation und ihre anatomischen Grundlagen. Ein neues Behandlungsprinzip in der Handchirurgie. Zur Behandlung der Spätstadien der Lunatummalacie und Navicularepseudarthrose. Hefte Unfallheilkd. 86, 1-109.

WILSON, J.N., 1973. Basal osteotomy of the first metacarpal in the treatment of arthritis of the carpometacarpal joint of the thumb. Br. J. Surg. 60, 854-8. https://doi.org/10.1002/bjs. 1800601106

WolF, J.M., DelaRONDE, S., 2012. Current trends in nonoperative and operative treatment of trapeziometacarpal osteoarthritis: a survey of US hand surgeons. J. Hand Surg. Am. 37, 77-82. https://doi.org/10.1016/j.jhsa.2011.10.010

WULle, C., 1993. Die Abductor pollicis longus-Plastik zur Behandlung der Daumensattelgelenkarthrose. Handchir Mikrochir Plast Chir. 25, 250-5.

Xu, L., Strauch, R.J., Ateshian, G.A., Pawluk, R.J., Mow, V.C., Rosenwasser, M.P., 1998. Topography of the osteoarthritic thumb carpometacarpal joint and its variations with regard to gender, age, site, and osteoarthritic stage. J. Hand Surg. Am. 23, 454-64. https://doi.org/10.1016/S0363-5023(05)80463-0

YAO, J., PARK, M.J., 2008. Early treatment of degenerative arthritis of the thumb carpometacarpal joint. Hand Clin. 24, 251-61, v-vi. https://doi.org/10.1016/j.hcl.2008.03.001

Zhang, W., Doherty, M., Leeb, B.F., Alekseeva, L., Arden, N.K., Bijlsma, J.W., Dincer, F., Dziedzic, K., Hauselmann, H.J., Kaklamanis, P., Kloppenburg, M., Lohmander, L.S., Maheu, E., MartinMola, E., PAVelka, K., Punzi, L., Reiter, S., Smolen, J., Verbruggen, G., Watt, I., ZimmermannGORSKA, I., 2009. EULAR evidence-based recommendations for the diagnosis of hand osteoarthritis: report of a task force of ESCISIT. Ann. Rheum. Dis. 68, 8-17. https://doi.org/10.1136/ard.2007.084772

Zschöck-Holle, A., Reik, M., Wölfle, O., Sauerbier, M., 2015. Behandlung der Rhizarthrose mittels Trapezium-Resektion und Swanson-Silikonprothese. Handchir Mikrochir Plast Chir. 47, 7-16. https://doi.org/10.1055/s-0034-1398667

Zumhasch, R., WagneR, M., KLAusch, S., 2012. Anatomie und Biomechanik der Hand. Georg Thieme Verlag, Stuttgart/New York, 49-59 


\section{Originalpublikation}

https://www.thieme-connect.de/products/ejournals/abstract/10.1055/a-0900-4130

DOI: $10.1055 / a-0900-4130$

\section{Literatur:}

1. Armstrong AL, Hunter JB, Davis TR. The prevalence of degenerative arthritis of the base of the thumb in post-menopausal women. J Hand Surg Br 1994; 19: 340-341, DOI: 10.1016/0266-7681(94)90085-X

2. Eaton RG, Littler JW. Ligament reconstruction for the painful thumb carpometacarpal joint. J Bone Joint Surg Am 1973; 55: 1655-1666, DOI: 10.2106/00004623197355080-00010

3. Fontana L, Neel S, Claise JM et al. Osteoarthritis of the thumb carpometacarpal joint in women and occupational risk factors: a case-control study. J Hand Surg Am 2007; 32: 459-465, DOI: 10.1016/j.jhsa.2007.01.014

4. Manara M, Bortoluzzi A, Favero M et al. Italian Society for Rheumatology recommendations for the management of hand osteoarthritis. Reumatismo 2013; 65: 167-185, DOI: $10.4081 /$ reumatismo.2013.167

5. Botha-Scheepers S, Riyazi N, Watt I et al. Progression of hand osteoarthritis over 2 years: a clinical and radiological follow-up study. Ann Rheum Dis 2009; 68: 12601264, DOI: 10.1136/ard.2008.087981

6. Riyazi N, Rosendaal FR, Slagboom E et al. Risk factors in familial osteoarthritis: the GARP sibling study. Osteoarthritis Cartilage 2008; 16: 654-659, DOI: 10.1016/j.joca.2007.10.012

7. Jensen V, Boggild $\mathrm{H}$, Johansen JP. Occupational use of precision grip and forceful gripping, and arthrosis of finger joints: a literature review. Occup Med (Lond) 1999; 49: 383-388, DOI: 10.1093/occmed/49.6.383

8. Dahaghin S, Bierma-Zeinstra SM, Ginai AZ et al. Prevalence and pattern of radiographic hand osteoarthritis and association with pain and disability (the Rotterdam study). Ann Rheum Dis 2005; 64: 682-687, DOI: 10.1136/ard.2004.023564 
9. Zhang W, Doherty M, Leeb BF et al. EULAR evidence based recommendations for the management of hand osteoarthritis: report of a Task Force of the EULAR Standing Committee for International Clinical Studies Including Therapeutics (ESCISIT). Ann Rheum Dis 2007; 66: 377-388, DOI: 10.1136/ard.2007.084772

10. Mentzel M, Ebinger T, Heckmann E et al. Ergebnisse nach Rhizarthrosebehandlung - Vergleich der Aufhängeplastik nach Epping mit der alleinigen Trapezektomie. Handchir Mikrochir Plast Chir 2001; 33: 176-180, DOI: 10.1055/s-2001-15127

11. Bakri K, Moran SL. Thumb carpometacarpal arthritis. Plast Reconstr Surg 2015; 135 : 508-520, DOI: 10.1097/PRS.0000000000000916

12. Nylen S, Juhlin LJ, Lugnegard H. Weilby tendon interposition arthroplasty for osteoarthritis of the trapezial joints. J Hand Surg Br 1987; 12: 68-72, DOI: 10.1016/02667681(87)90062-3

13. Epping W, Noack G. Die operative Behandlung der Sattelgelenksarthrose. Handchir Mikrochir Plast Chir 1983; 15: 168-176 PMID: 6629152

14. Sigfusson R, Lundborg G. Abductor pollicis longus tendon arthroplasty for treatment of arthrosis in the first carpometacarpal joint. Scand J Plast Reconstr Surg Hand Surg 1991; 25: 73-77, DOI: 10.3109/02844319109034926

15. Wulle C. Die Abductor pollicis longus-Plastik zur Behandlung der Daumensattelgelenkarthrose. Handchir Mikrochir Plast Chir 1993; 25: 250-255, PMID: 8144072

16. Katzel EB, Bielicka D, Shakir S et al. Midcarpal and Scaphotrapeziotrapezoid Arthritis in Patients with Carpometacarpal Arthritis. Plast Reconstr Surg 2016; 137: 17931798, DOI: 10.1097/PRS.0000000000002160

17. Warganich T, Shin AY. A Technique for the Management of Concomitant Scaphotrapezoid Arthritis in Patients With Thumb Metacarpotrapezial Arthritis: Interposition Arthroplasty With a Capitate Suture Anchor. Tech Hand Up Extrem Surg 2017; 21 : 71-74, DOI: 10.1097/BTH.0000000000000155

18. Eaton RG, Lane LB, Littler JW et al. Ligament reconstruction for the painful thumb carpometacarpal joint: a long-term assessment. J Hand Surg Am 1984; 9: 692-699, DOI: 10.1016/S0363-5023(84)80015-5

19. Burton RI, Pellegrini VD, Jr. Surgical management of basal joint arthritis of the thumb. Part II. Ligament reconstruction with tendon interposition arthroplasty. J Hand Surg Am 1986; 11: 324-332, DOI: 10.1016/S0363-5023(86)80137-X 
20. Wolf JM. Treatment of scaphotrapezio-trapezoid arthritis. Hand Clin 2008; 24: 301306, vii, DOI: 10.1016/j.hcl.2008.03.002

21. Outerbridge RE. The etiology of chondromalacia patellae. J Bone Joint Surg Br 1961; 43-B: 752-757, DOI: 10.1302/0301-620X.43B4.752

22. Noback PC, Lombardi JM, Seetharaman M et al. Development and Validation of a Disease-Specific Questionnaire for Basal Joint Arthritis. J Wrist Surg 2017; 6: 126 133, DOI: 10.1055/s-0036-1593612

23. Bijur PE, Silver W, Gallagher EJ. Reliability of the visual analog scale for measurement of acute pain. Acad Emerg Med 2001; 8: 1153-1157, DOI: 10.1111/j.15532712.2001.tb01132.x

24. Kapandji A. Cotation clinique de l'opposition et de la contre-opposition du pouce. Ann Chir Main 1986; 5: 67-73, DOI: 10.1016/s0753-9053(86)80053-9

25. Desrosiers J, Hebert R, Bravo G et al. Comparison of the Jamar dynamometer and the Martin vigorimeter for grip strength measurements in a healthy elderly population. Scand J Rehabil Med 1995; 27: 137-143, PMID: 8602475

26. Neumann S, Kwisda S, Krettek C et al. Comparison of the Grip Strength Using the Martin-Vigorimeter and the JAMAR-Dynamometer: Establishment of Normal Values. In Vivo 2017; 31: 917-924, DOI: 10.21873/invivo.11147

27. Wajon A, Vinycomb T, Carr E et al. Surgery for thumb (trapeziometacarpal joint) osteoarthritis. Cochrane Database Syst Rev 2015, DOI: 10.1002/14651858.CD004631.pub4

28. Sodha S, Ring D, Zurakowski D et al. Prevalence of osteoarthrosis of the trapeziometacarpal joint. J Bone Joint Surg Am 2005; 87: 2614-2618, DOI: 10.2106/JBJS.E.00104 


\section{Danksagung}

Mein Dank gilt all denen, durch deren Unterstützung diese Arbeit ermöglicht wurde.

Ich danke meinem Doktorvater Herrn Prof. Dr. med. Dr. h. c. Raymund E. Horch und meinem Betreuer Herrn PD Dr. med. Alexander D. Bach für das Vertrauen, die Unterstützung und allem voran die Möglichkeit, diese Arbeit zu verfassen. Ich danke ihnen für den stets hilfreichen und kompetenten Rat und die warmherzige und professionelle Begleitung. Insbesondere bin ich dankbar für die Freiheit, die sie mir während des Projektes gewährt haben.

Ein außerordentlicher Dank geht an alle Mitarbeiter und Mitarbeiterinnen der Klinik für Plastische und Ästhetische Chirurgie - Handchirurgie - Wiederherstellungschirurgie Eschweiler. Ohne ihre Unterstützung und Geduld wäre dies nicht möglich gewesen.

Mein herzlicher Dank gilt Dr. rer. pol. Mike Farjam für die statistische Beratung, meinen Geschwistern Marie-Anne, Constantin und Cornelius sowie meinen Freunden Herrn Dr. med. Viet-Tuan Huynh und Frau Dr. med. Isabelle Morgenstern für die Beratung, die Überarbeitung und die buchstäbliche Korrektur meiner Dissertation.

Rebecca Klein danke ich für die Standhaftigkeit, Ruhe und Geduld in den Phasen, in denen mich so manches Tief der Verzweiflung nahebrachte und sie mir mit Verständnis und Zuspruch zur Seite stand.

Meinen Eltern gilt mein innigster Dank. Sie ermöglichten mir das Studium der Humanmedizin und machten mich durch ihre immerwährende Liebe und bedingungslose Fürsorge maßgeblich zu dem Menschen, der ich heute bin. Es gibt keine Worte, die meinen Dank dafür adäquat ausdrücken könnten, was sie für meine Geschwister und mich zu leisten bereit waren und sind. 